\title{
Consecuencias de la intrusión marina en la España mediterránea e insular según el informe SASMIE
}

\author{
Emilio Custodio \\ Real Academia de Ciencias. Departamento de Ingeniería Civil y Ambiental, Universidad Politécnica \\ de Cataluña, Campus Nord. C/ Jordi Girona 1-3, Edificio D2(007), 08034 Barcelona. \\ emilio.custodio@upc.edu
}

\begin{abstract}
RESUMEN
Desde hace décadas se conoce la salinización de los acuíferos costeros españoles mediterráneos e insulares. Sólo en unos pocos casos esta situación está bien gestionada, ya que afecta a pequeñas extensiones de territorio y a derechos de agua, muchos de los cuales siguen siendo privados en la práctica. La salinización del agua subterránea afecta a muchos y a numerosas actividades industriales, turísticas y agrícolas. Los mayores problemas aparecieron en las últimas décadas del siglo XX. Parte de ellos aún persisten, pero otros han ido decreciendo a medida que se ha puesto a disposición agua de otros orígenes. El abandono de parte de los acuíferos es una importante pérdida patrimonial y una disminución de la garantía de disponibilidad de agua si no se introducen otras actuaciones, que suelen ser más agresivas ambientalmente. El cambio global y climático puede modificar esta situación. Los acuíferos costeros pueden ser recuperados si el coste es proporcionado. La intrusión marina supone un deterioro del bienestar, debido a la mayor corrosión, efecto negativo en la agricultura, empeoramiento del estado de humedales y merma de la descarga de agua dulce al mar, que es ecológicamente importante. Todo esto va más allá del buen estado del acuífero que exige la Directiva Marco del Agua europea. Se requieren actuaciones a largo plazo, tanto estructurales como no estructurales. La experiencia existente en España se ha sintetizado en el informe SASMIE (2017), realizado por la Universidad Politécnica de Cataluña con el apoyo de SUEZ-España y la supervisión de CETAQUA, que se centra en la parte mediterránea e insular española. Se presentan algunas de las constataciones más destacadas, según los datos disponibles.
\end{abstract}

Palabras clave: acuíferos costeros, España mediterránea, gestión, intrusión marina, islas.

\section{Results of seawater intrusion in Mediterranean Spain and the Spanish islands according to the SASMIE report}

\begin{abstract}
Groundwater salinization of the Spanish aquifers by seawater has been known for decades. However, in only a few cases has this been managed to control the damage, as salinization affects small territories and involves water rights that in practice remain largely in the private domain. Groundwater salinization affects many people and industrial, tourist and agricultural activities. The greatest problems appeared in the last decades of the 20th century. Some of them still persist but others have been dwindling as water from other origins rather than local ones has been made available to substitute existing pumped wells. The abandonment of part of the coastal aquifers as a freshwater resource is an important hydraulic inheritance loss and especially a decrease in the guarantee of water availability if other costly and environmentally less friendly structures are not incorporated. Global and climatic change may modify the current situation. Coastal aquifers can be recovered if the cost is proportionate. Seawater intrusion implies an impairment to general wellbeing due to enhanced corrosion, negative effects on agriculture, deterioration of wetlands, and a decrease in the ecologically important continental freshwater outflow to the ocean. All this goes beyond the scope of the aquifer good status of coastal groundwater bodies to be achieved according to the European Water Framework Directive. Long-term manag ement action is needed, both structural and non-structural. The current experience in Spain has been synthetized in the SASMIE (2017) report, developed by the Technical University of Catalonia with the support of SUEZ-Spain and the overview of CETAQUA, which centers on the Mediterranean and island areas of Spain. Some of the most relevant situations here are considered, based on the available data.
\end{abstract}

Keywords: coastal aquifers, islands, management, Mediterranean Spain, seawater intrusion. 


\section{Peculiaridades de los acuíferos costeros}

Aquí se consideran acuíferos costeros aquellos que tienen contacto lateral o por superposición con el mar, aunque su extensión hacia el interior del continente o isla sea dominante. Estos acuíferos son susceptibles de contener naturalmente o de forma inducida agua salina resultante de la presencia de agua de origen marino en alguna porción del mismo. El agua marina, que es más densa que el agua dulce, penetra en el continente o isla, desde escasos metros hasta varios $\mathrm{km}$, en función de las características hidrogeológicas (espesor de las formaciones permeables y su conductividad hidráulica) y del caudal de descarga de agua continental en la costa.

Los principios básicos fueron ya bien establecidos a principios del siglo $X X$ y después se desarrollaron a partir de la década de 1950, cuando se asentaron los conceptos, métodos de cálculo y tratamiento cuantitativo, procesos hidrogeoquímicos e isotópicos ambientales (Custodio, 1976; Custodio y Bruggeman, 1987; Werner et al., 2013), se avanzó en el tratamiento de los fluidos de densidad variable (Carrera et al., 2011) y se elaboraron los principios de gestión (Bear, 2007).

El interés por la salinización de acuíferos costeros viene de tiempos ancestrales, en especial a lo largo de la costa del mar Mediterráneo y algunas islas, pero se trata de descripciones o de intentos de interpretar las observaciones en carbonatos costeros karstificados por los filósofos griegos; en parte, esas interpretaciones desenfocadas retrasaron el inicio de la hidrogeología como rama de la ciencia. A pesar de la importancia real de los acuíferos costeros, el número de casos bien estudiados es relativamente escaso, aunque creciente. Una recopilación es la de Post y Abarca (2010).
La intrusión marina en acuíferos costeros es un fenómeno natural. El tránsito entre el agua dulce y el agua marina puede ser brusco, aunque generalmente se tiene una zona de mezcla cuyo espesor es muy variable según las condiciones hidrogeológicas generales y con gran importancia de las heterogeneidades locales. El clásico esquema de la existencia de una cuña de intrusión marina que penetra y se adelgaza hacia el interior del continente o isla ilustra bien las situaciones de medios homogéneos y ayuda a asentar conceptos. Sin embargo, la realidad en acuíferos heterogéneos, que es lo más común, es mucho más compleja, en especial a nivel del detalle necesario para los trabajos prácticos. Esto es especialmente cierto en sedimentos costeros detríticos y en carbonatos karstificados. La suficiente buena comprensión requiere un conocimiento hidrogeológico y piezométrico en tres dimensiones. Este es uno de los principales retos a afrontar y que requiere métodos adecuados para conocer y cuantificar los procesos involucrados y que tengan en cuenta que las situaciones pueden cambiar notablemente a lo largo del tiempo.

La designación intrusión marina hace referencia tanto a la penetración del agua marina en las formaciones continentales de forma natural, como a la progresiva mayor penetración por causas antrópicas, cambio climático y/o ascenso eustático.

La descarga de agua dulce o salobre a lo largo de la costa tiene importancia para la existencia y características peculiares de los humedales costeros y de las circunstancias de las aguas marinas litorales junto a la costa. Esa descarga crea determinadas condiciones de salinidad, composición química y contenido en nutrientes, que son importantes ecológicamente y que se asocian a servicios económicos de notable interés
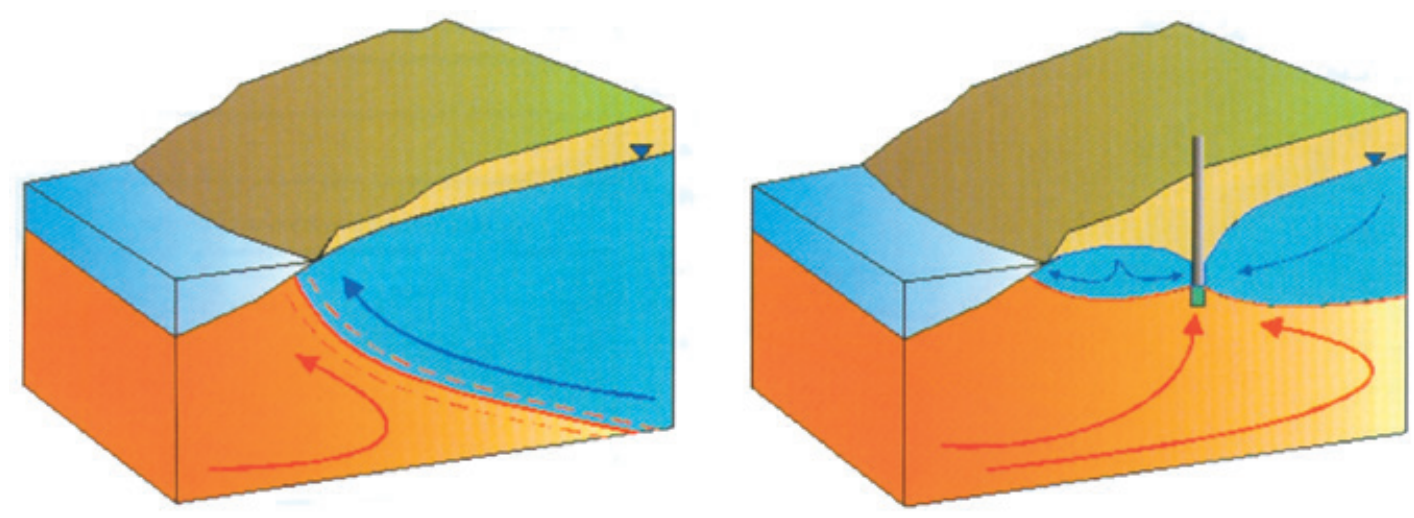

Figura 1. Flujo de agua dulce y agua salada en una sección de un acuífero costero libre idealizado con recarga y en estado natural (figura izquierda) y afectado por la extracción de un pozo (figura derecha) (Pool et al., 2007).

Figure 1. Freshwater and saline water flow in the cross-section of an idealized coastal aquifer, recharged and under natural conditions (left figure) and affected by a pumping well (right figure) (Pool et al., 2007). 
social. El caudal de descarga al mar puede ser notablemente mayor que la descarga continental o insular, ya que a dicho caudal se adiciona el flujo de agua marina reciclado dinámicamente, que puede incrementarse por efecto de marea (Figura1).

Las extracciones de agua subterránea de un acuífero costero reducen la descarga al mar, con retraso y amortiguación respecto a los cambios que se producen en dichas extracciones. El resultado de un aumento de las extracciones es una progresiva disminución de la descarga de agua dulce al mar, acompañada de un lento avance en el continente o isla de la cuña de intrusión marina y, en general, una expansión de la zona de mezcla entre el agua dulce y el agua salada, en función de las heterogeneidades existentes. En el caso de pozos y otras obras de captación de agua subterránea en que bajo los mismos se encuentra agua salada o salina en profundidad, el descenso de niveles que crea la extracción o drenaje favorece el ascenso de la zona de mezcla y que el agua captada pueda llegar a salinizarse progresivamente con cierta rapidez. La mezcla con un 2 o $3 \%$ de agua marina hace que el agua extraída resulte inapropiada para la mayoría de usos.

Todos estos aspectos son bien conocidos y están desarrollado en numerosos libros básicos de hidrogeología y de hidrología subterránea, pero frecuentemente no suelen formar parte del bagaje de conocimiento habitual de expertos en hidrogeología y en la explotación y gestión del agua subterránea. Con frecuencia, estos expertos no tienen en cuenta la importancia que tiene el flujo tridimensional del agua subterránea de densidad variable y el papel dominante que pueden tener las heterogeneidades. Por otro lado, las circunstancias locales son frecuentemente pobremente conocidas ya que requieren estudios y observaciones mucho más pormenorizadas de lo que es común en hidrogeología regional. Para deducir los detalles a partir de información escasa, dispersa y no focalizada se requiere de expertos. Los perfiles de salinidad de sondeos rara vez representan lo que realmente sucede en el acuífero y son origen de frecuentes malinterpretaciones y de que se tomen decisiones erróneas.

Para las autoridades del agua, muchas veces la parte de un acuífero costero afectable por intrusión marina es sólo una porción y el acuífero costero es además una parte pequeña del territorio que deben gestionar. Por esta razón tienden a no prestar la debida atención a la problemática de la intrusión marina y buscan paliar los problemas derivados de la salinización en abastecimientos y regadíos mediante aducción de otras fuentes de agua alternativas. Estas pueden y suelen ser más costosas y conflictivas y menos aceptables medioambientalmente, pero más dóciles y tratables con visión de ingeniería clásica.

\section{Acuíferos costeros mediterráneos españoles: Informe SASMIE}

España tiene una larga costa a lo largo de la cual se encuentran diferentes tipos de acuíferos bajo distintas condiciones. La explotación a caudales significativos de estos acuíferos costeros se inició a finales del siglo XIX en el entorno de Barcelona, fue ya intensiva en la década de 1940 en el área del delta del río Besós y Llano de Barcelona y en la década de 1960 en el delta del río Llobregat. Se trata de un caso paradigmático y por eso se desarrolla con mayor detalle, ya que permite entender situaciones menos documentadas. Después de algunos años se produjo la salinización de parte de los acuíferos profundos, los más explotados, y se abandonaron numerosos pozos de abastecimiento a población y a industrias. Esto llevó a que el hoy extinto Servicio Geológico de Obras Públicas (SGOP) del Ministerio de Obras Públicas y la Comisaría de Aguas del Pirineo Oriental iniciasen estudios de detalle, con el apoyo de la Universidad Politécnica de Cataluña y la Sociedad General de Aguas del Barcelona. Los estudios se extendieron después a otras áreas del Pirineo Oriental (hoy Distrito Fluvial de Cataluña). La actividad del SGOP se expandió también a otros acuíferos peninsulares mediterráneos (por ejemplo, los de la provincia de Castelló), a las Islas Baleares y en especial a las Islas Canarias dentro del proyecto PNUD-Gobierno español conocido como SPA-15 (1975). También se realizaron otros estudios de cierto detalle en otras áreas mediterráneas españolas por el Instituto Geológico y Minero de España (IGME, que durante algunos años se designó como Instituto Tecnológico GeoMinero de España, ITGE). En esas épocas se produjo un notable avance en el conocimiento a partir de estudios especialmente orientados y apoyados en redes de observación.

Las primeras indicaciones sobre la importancia de la intrusión marina en España y sus consecuencias son de la década de 1960, referidas al delta del Llobregat (Barcelona) (Custodio 1967), después ampliadas a otras circunstancias en una sección de un extenso libro (Custodio, 1976) y en comunicaciones internacionales (Custodio, 1981b; 1987). El IGME recopiló numerosos datos en sus estudios de acuíferos dentro del proyecto PIAS (Plan de Investigación de Aguas Subterráneas) y otros llevados a cabo en las décadas de 1970 y 1980, lo que permitió presentar una figura resumen (MOPTMA-MINER, 1994), que después evolucionó a lo que muestra la Figura 2, tomada del Libro Blanco del Agua (MIMAM, 2000). Una versión más reciente es la de la Figura 3. En la Tabla 1 se indica el número de las MASb (masa de agua subterránea) que tienen problemas de intrusión marina, según los planes hidrológicos. Uno 


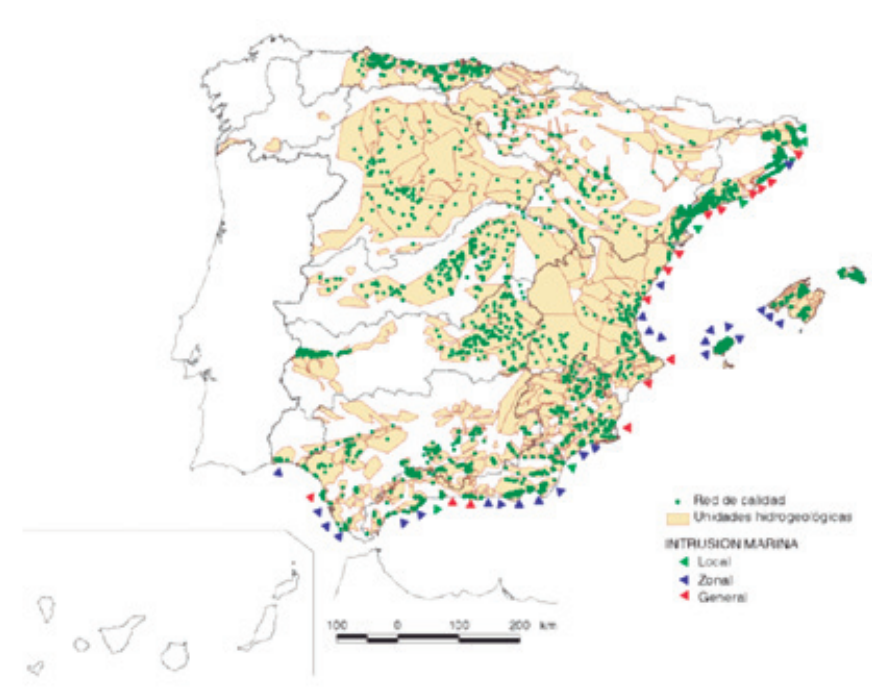

Figura 2. Red de observación de la calidad de las aguas subterráneas en España en 1999, según MIMAM (2000), con indicación de dónde existen problemas de intrusión marina.

Figure 2. Monitoring network of groundwater quality in Spain in 1999, according to MIMAM (2000), showing where there are seawater intrusion problems.

de los primeros resúmenes de la situación general fueron los de Custodio (1981a), después actualizados en López-Geta y Gómez-Gómez (2007).

En el último tercio del siglo $X X$ se produjeron serios problemas de salinización de acuíferos en ciertas áreas, que repercutieron fuertemente en los abastecimientos urbanos, turísticos e industriales. Tal sucedió en el entorno deTarragona, entre Calafell y Salou, y también en Mallorca y Eivissa (Ibiza) en las Islas Baleares y en el Este de Gran Canaria, en las Islas Canarias. En determinadas poblaciones y momentos, el agua disponible para abastecimiento urbano llegó a salinidades altas, de modo que no era posible su utilización, excepto para servicios sanitarios y refrigeración industrial.

La intrusión marina y los problemas de las áreas costeras han sido tratados en diversas reuniones técnico-científicas, como los sucesivos Simposios sobre Tecnología de la Intrusión Marina (TIAC), promovidos por el IGME, en las que se aportaba una revisión de la situación española, como la de Gómez Gómez et al. (2003). España ha organizado dos reuniones internacionales sobre la intrusión marina, como parte de las Salt Water Intrusion Meeting (SWIM), en 1992 (BarceIona, XII SWIM) y en 2004 (Cartagena, XVIII SWIM), con aportaciones a muchas de las otras reuniones SWIM en otros lugares y que se puede consultar en la web de dicho grupo (http://www.swim-site.nl/proceedings). EI IGME (2000) editó informes sobre la situación en el área mediterránea española. No obstante, como suce- de también en otros países, sólo se dispone de unos pocos casos españoles de estudio detallado de acuíferos costeros. En los otros acuíferos el conocimiento frecuentemente se reduce a descripciones sucintas y reconocimientos preliminares, a veces con constataciones y deducciones no fundamentadas y aun erróneas.

Para reunir y discutir la experiencia existente en la costa mediterránea española y de los archipiélagos Balear y de Canarias, se ha realizado el informe SASMIE (2017). SASMIE es el acrónimo de Salinización de las aguas subterráneas en los acuíferos costeros mediterráneos e insulares españoles. El objetivo fue el de disponer de una recopilación no exhaustiva, pero lo más completa posible, de los problemas de intrusión marina en las áreas indicadas, lo que excluye la parte peninsular atlántica española, donde se encuentran Doñana y el litoral de Huelva. Además de presentar y exponer los principios básicos y de detalle, se consideran también los aspectos ambientales, económicos, de gestión y sociales, con sus logros y fallos. La recopilación se ha limitado a lo que está disponible en forma escrita accesible y se ha complementado con entrevistas y cuestionarios a expertos y gestores. La realización se ha amparado en un acuerdo entre la Universidad Politécnica de Cataluña (UPC, Grupo de Hidrología Subterránea del Departamento de Ingeniería Civil y Ambiental) y SUEZ-Solutions (actualmente SUEZ España), con el apoyo de CETAQUA (CentroTecnológico del Agua, de SUEZ España). El informe resultante ha sido publicado como un e-book de libre acceso por la UPC (SASMIE, 2017). Dicho informe utiliza datos precedentes del informe MASE (2015) en el caso del Levante (Nordeste) español y Canarias.

Lo que se aporta a continuación son los resultados más destacados y que puedan ser de utilidad para los estudios de conocimiento, observación y gestión de los acuíferos costeros españoles. Por esta razón lo que se expone no tiene la estructura de un trabajo de investigación y no contiene nuevas aportaciones más allá que las de una recopilación razonada y sintetizada, que busca lo que hay de común. En el informe SASMIE se aportan las referencias más importantes, las que permiten conocer lo que se ha hecho previamente con carácter no de detalle y así poder partir de lo que se ha logrado anteriormente.

\section{Consideraciones generales sobre la intrusión marina en España}

Aunque las formaciones costeras son hidrogeológicamente muy variadas, a lo largo de la costa mediterránea española hay una tendencia a dominar unas sobre otras, lo que permite cierta tipificación. 


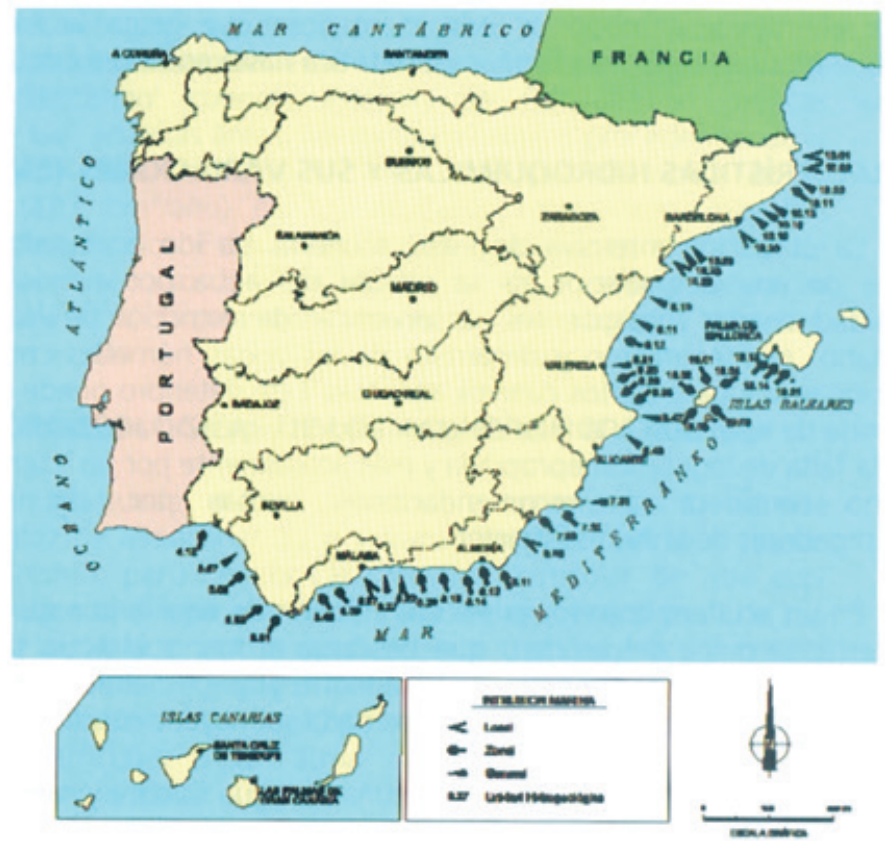

Figura 3. Actualización de los acuíferos costeros españoles con problemas locales, zonales y generales (según la simbología) en relación con la intrusión marina, según López-Geta y Fernández Ruiz (2012). Se indica el número de la MASb (masa de agua subterránea) correspondiente. La calidad reprográfica es la disponible en la referencia.

Figure 3. Updating of Spanish coastal aquifers with local, areal and generalized problems (according to the symbols) related with seawater intrusion, from López-Geta and Fernández-Ruiz (2012). The corresponding groundwater body number is indicated. The reprographic quality is that available in the reference.

Al norte de Barcelona dominan los acuíferos costeros en pequeños aluviales y llanos (planas). Alrededor de Barcelona las principales formaciones costeras son los deltas Plio-Cuaternarios del Besós y en especial del Llobregat y sus aluviales y depósitos litorales. Al sur de Barcelona y hasta el sur de Peníscola (Peñíscola)-Serra d'Irta dominan los carbonatos Mesozoicos y las calcarenitas Miocenas y se encuentra el delta del Ebre (Ebro). Al sur de Peníscola dominan las planas Cuaternarias y Plio-Miocenas costeras estrechas y alargadas según el litoral, hasta Dénia. Más hacia el sur, en la provincia de Alacant (Alicante), muchos de los acuíferos costeros consisten en macizos carbonatados karstificados que evolucionan hacia grandes bloques muy productivos, pero relativamente aislados, y que forman parte de una masa caótica poco permeable que está asociada a las partes distales de las formaciones de la cordillera Bética tras la orogenia alpina. Además, se encuentran sedimentos detríticos que rellenan las fosas de origen tectónico. Al oeste de los importantes acuíferos costeros en carbonatos del Campo de Dalías-Sierra de Gádor, los acuíferos costeros son en buena parte pequeños depósitos aluviales y costeros, hasta el Campo de Gibraltar, aunque pueden tener gran importancia local.

En las Islas Baleares dominan los carbonatos Mesozoicos y las calcarenitas mioceno-pliocenas, que en ocasiones pueden estar notablemente karstificadas. En las Islas Canarias todas las formaciones costeras son volcánicas o asociadas al volcanismo. Esto incluye conos de deyección y pequeños deltas, así como rellenos lávicos relativamente recientes que han movido la línea de costa mar adentro.

Los distintos deltas a lo largo de la costa mediterránea española tienen una estructura sedimentaria similar, de relleno del espacio litoral (Figura 4), aunque sus características hidrogeológicas pueden diferir notablemente en función de la granulometría de los materiales y la profundidad del substrato en la costa. Son el resultado de la gran elevación eustática desde finales del Pleistoceno, hace unos 11.000 años, tras la última glaciación (Custodio, 2010), junto a los procesos litorales de deriva de sedimentos.

El delta del Ebre es un clásico delta de un río de gran porte, que contiene agua marina en el acuífero cautivo tardi-pleistoceno profundo a causa de la muy pequeña altitud en el lugar de tránsito al valle bajo (Figura 5). Esta existencia de agua marina antigua en este delta y otros lugares de la costa española ha sido estudiada por Manzano et al. (2001).

En los otros deltas mediterráneos españoles el agua marina original ha podido ser expulsada en buena parte del acuífero profundo, ya que la elevación del cauce fluvial en el vértice del delta (lugar de contacto entre el delta y el valle bajo) es suficientemente alta a causa de la elevada pendiente de ríos que tienen un marcado carácter torrencial y con sedimentos groseros (Figura 6). Se supone que el acuífero profundo aflora o subaflora en el fondo marino, mar adentro, como está bien constatado para el delta del Llobregat (Figura 7). El delta de LaTordera, junto a la población de Blanes, difiere del delta del Llobregat en que en éste el acuitardo intermedio (formación fina entre el acuífero profundo y las actuales formaciones deltaicas someras) tiene muy baja permeabilidad y en LaTordera es mayor por tratarse de sedimentos de aguas con mayor energía. También difiere el delta del Besós, en el mismo lado norte del llano de Barcelona, ya que el río en los momentos de menor nivel del mar no fue capaz de excavar completamente los materiales preexistentes donde actualmente está la línea de costa. Todo esto condiciona el funcionamiento. Por lo tanto, su conocimiento detallado es clave para entender el funcionamiento natural y bajo explotación y para definir la forma de intrusión marina y cómo gestionarla. Esto muestra que similitudes aparentes no responden a comportamientos comparables. 


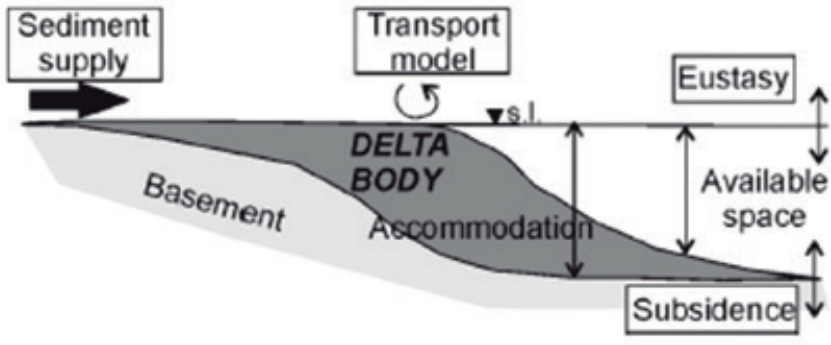

Figura 4. Esquema de formación de un espacio deltaico, según Rabineau (2001).

Figure 4. Diagram of the formation of a free deltaic space, from Rabineau (2001).

La realidad es notablemente más compleja que lo se muestra en las figuras anteriores (véase la Figura 8, que corresponde al delta del Llobregat), pero las simplificaciones explican los grandes rasgos de la intrusión marina si se considera que los detalles pueden englobarse como una gran dispersividad hidrodinámica. Los acuíferos cautivos profundos tardi-pleistocenos que se esquematizan en las Figuras 6 y 7 no son un continuo homogéneo, sino que presentan variaciones asociadas a los antiguos paleocauces del rio y de las rieras laterales, con depósitos eólicos, de llanura de inundación y litorales entre ellos.

La penetración progresiva de la intrusión marina en el delta del Llobregat (Figuras 9 y 10) a consecuencia de las extracciones de agua subterránea en el valle bajo y delta se realiza por caminos preferentes que se corresponden con los paleovalles y sedimentos grose- ros de borde. Esto se reproduce bien en la modelación numérica hidrogeológica (Vázquez-Suñé et al., 2006). Se tiene un seguimiento periódico de la evolución de la salinización de los acuíferos profundos. La Figura 11 muestra la situación en 2014.

Como se detalla en SASMIE (2017), los estudios relativos a la intrusión marina más desarrollados corresponden a los acuíferos profundos del delta del Llobregat. Otros casos con estudios de detalle, al menos de determinados aspectos, son los realizados en el acuífero de la Vall d'Uixó-Moncofa (provincia de Castelló) (García-Menéndez et al., 2016; Renau-Pruñonosa et al., 2016) en relación con el uso para regadío, en el delta del Andarax (junto a Almería) (Pulido-Bosch et al., 2002) para obtener agua marina del acuífero para alimentar a una gran planta de desalinización y en el Campo de Dalías-Sierra de Gádor (Figuras 12 y 13) para abastecer a la muy extensa área de invernaderos. Algunos de los pequeños aluviales de la provincia de Granada tienen importancia local y se han estudiado con cierto detalle (Calvache y Pulido-Bosch, 1997; Calvache et al., 2007).

No hay estudios de detalle en las Islas Canarias, salvo los realizados en relación con la creciente salinización en el Este de Gran Canaria (acuífero de Telde-Vecindario) (Cabrera y Custodio, 2004; 2005) y los llevados a cabo para determinar el origen del agua subterránea salobre en el SE de Gran Canaria (macizo de Amurga) (Custodio, 1990; 1992) y en la franja central de la isla de Fuerteventura (Herrera y Custodio, 2003).

Las costas del mar Mediterráneo contienen numerosos manantiales kársticos (Margat, 2004; Margat y van der Gun, 2013; Tulipano et al., 2003), como los

\begin{tabular}{|l|c|c|c|c|c|}
\hline $\begin{array}{l}\text { Demarcación } \\
\text { hidrográfica }\end{array}$ & Total MASb costeras & Sin intrusión marina & $\begin{array}{c}\text { Con intrusión } \\
\text { marina local }\end{array}$ & $\begin{array}{c}\text { Con intrusión } \\
\text { marina zonal }\end{array}$ & $\begin{array}{c}\text { Con intrusión } \\
\text { marina general }\end{array}$ \\
\hline Norte & 10 & 10 & 0 & 0 & 0 \\
\hline Guadiana & 1 & 0 & 0 & 1 & 0 \\
\hline Guadalquivir & 5 & 2 & 0 & 2 & 1 \\
\hline Sur & 19 & 9 & 4 & 4 & 2 \\
\hline Segura & 6 & 2 & 2 & 1 & 1 \\
\hline Júcar & 10 & 0 & 1 & 7 & 2 \\
\hline Ebro & 1 & 0 & 0 & 1 & 0 \\
\hline Cataluña & 13 & 2 & 4 & 1 & 6 \\
\hline Baleares & 23 & 14 & 0 & 5 & 4 \\
\hline Canarias & 7 & 0 & 0 & 7 & 0 \\
\hline Total & 95 & 39 & 11 & 29 & 16 \\
\hline
\end{tabular}

Tabla 1. Número de masas de agua subterránea (MASb) costeras en cada demarcación hidrográfica y su estado respecto a la intrusión marina en el entorno de 2010 (López-Geta y Fernández Ruiz, 2012).

Table 1. Number of coastal groundwater bodies (MASb) in each water district and their state relative to seawater intrusion in about 2010 (López-Geta y Fernández Ruiz, 2012). 
del sur de Francia, sur de Italia, Croacia y Eslovenia, Grecia y sus grandes islas, Turquía, Siria y Líbano, etc. Son algo muy característico y quizás uno de los aspectos que han acaparado más la atención desde la antigüedad. También existen en la parte española, aunque no son tan caudalosos debido a las menores y menos pluviosas cuencas de alimentación. Los manantiales costeros españoles más importantes están en la Serra d'Irta (manantiales de Peníscola, Badum y Alcossebre, en la provincia de Castelló) y en el área de Benissa-Calp (manantiales de El Moraig y Toix, en la provincia de Alacant). Otras descargas costeras son las del macizo de Garraf (manantiales de La Falconera y Aigua Dolç, al sur de Barcelona), en el macizo de Vandellós (en la provincia de Tarragona) y diversos lugares del Campo de Dalías (en la provincia de Almería, hoy agotados a causa de las extracciones de agua subterránea, como el manantial de Agua Dulce). Hay diversos manantiales costeros alrededor de la isla de Mallorca, como el de S'Almadrava, en el que se llega a descargar agua salobre por encima del nivel del mar. Ninguno de estos manantiales costeros tiene hoy aprovechamiento humano. Desde finales del siglo XIX se hicieron obras para intentar la captación en La Falconera, sin éxito. Hay algunas propuestas para EI Moraig, que no han pasado de simples bocetos.

Los estudios más completos realizados en los acuíferos costeros españoles han dispuesto de perforaciones de estudio y combinan las consideraciones hidrodinámicas con las hidrogeoquímicas y los isótopos ambientales. En general son el fruto de una simbiosis entre los medios y capacidades de la administración del agua y de los institutos públicos de estudio antes mencionados, con grupos especializados en algunos departamentos universitarios, como los de las Universidades Politécnica de Cataluña (UPC), Jaume I de Castelló, Granada, Almería, Las Palmas de Gran Canaria y Alacant. Sin embargo, dicha actividad y colaboración ha decaído notablemente en las últimas dos décadas, así como las observaciones. Actualmente se desarroIlan estudios avanzados y teóricos en el IDAEA-CSIC de Barcelona, conjuntamente con la UPC, dentro del Grupo de Hidrología Subterránea. Estos estudios han incorporado recientemente una pequeña área experimental costera (Proyecto Medistraes) en el acuífero de la parte inferior de la riera de Argentona (Barcelona).

\section{Consideraciones ambientales}

Se pueden considerar cuatro aspectos principales ambientales en relación con la explotación de los acuíferos costeros.

1) Humedales costeros que dependen del agua subterránea, en los que los gradientes de salinidad y las fluctuaciones juegan un papel importante, así como el aporte de nitrato. Este es el caso del humedal de S'Albufera (en el NE de Mallorca) y en las lagunas y áreas encharcadas del delta del Ebre;

2) Papel de la descarga de agua subterránea en el agua marina litoral y la creación de condiciones especiales de salinidad, hidroquímica y aporte de nutrientes. Un caso paradigmático es el del Mar Menor,

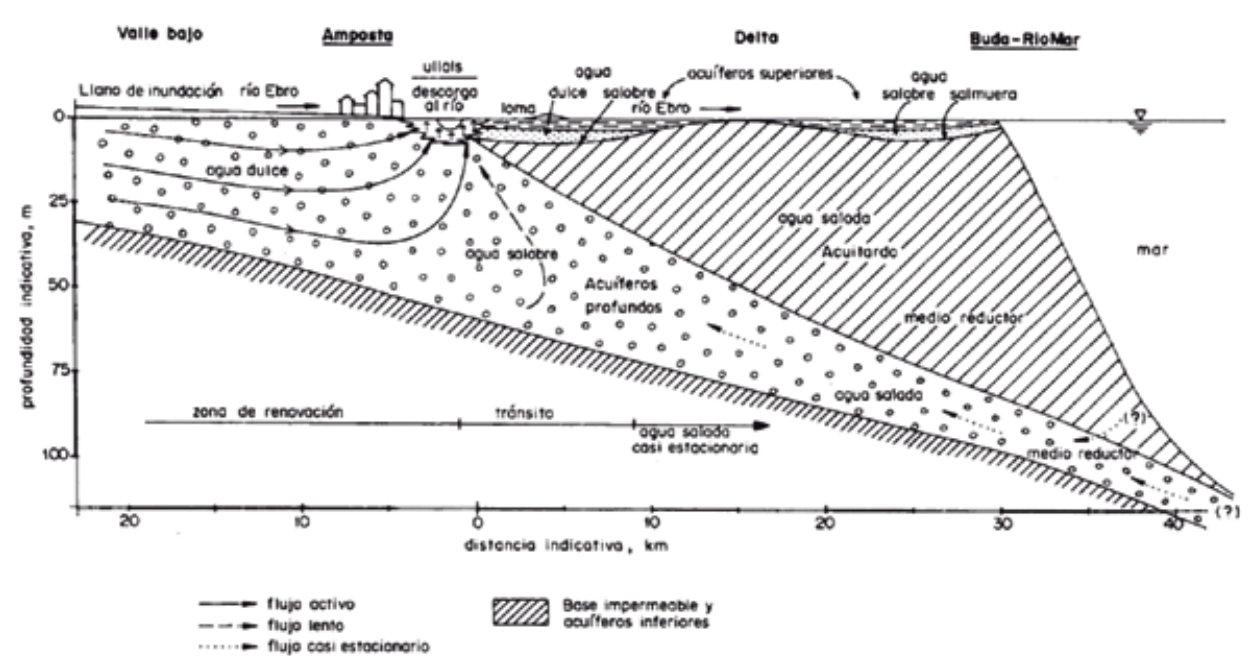

Figura 5. Esquema hidrogeológico longitudinal del delta del Ebre y del flujo del agua subterránea (Bayó et al., 1992; 1997).

Figure 5. Longitudinal hydrogeological scheme of the Ebre delta and of groundwater flow (Bayó et al., 1992; 1997). 


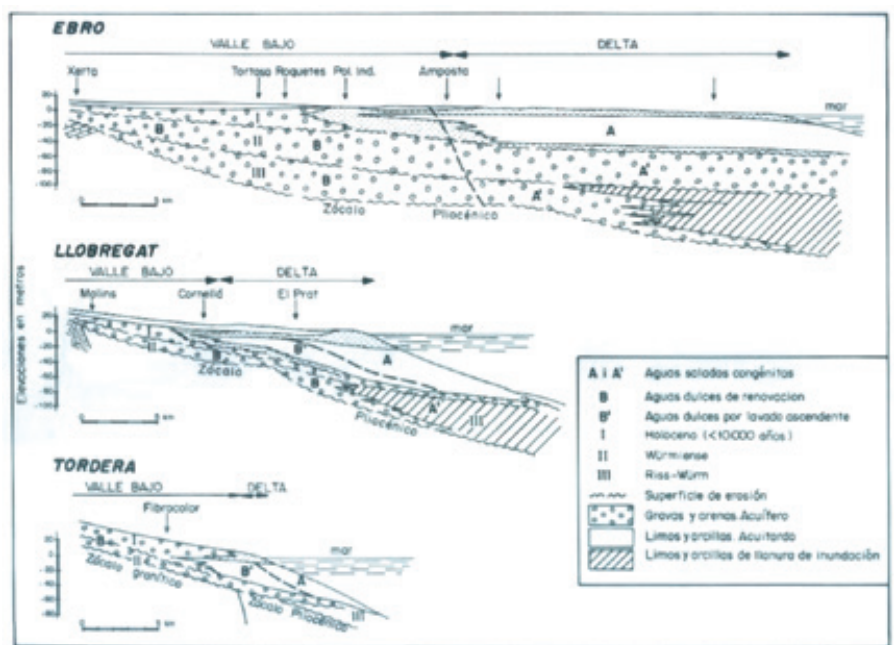

Figura 6. Esquemas simplificados de la estructura sedimentaria de los deltas de los ríos Ebre, Llobregat y La Tordera (Bayó y Custodio, 1989).

Figure 6. Simplified diagrams of the sedimentary structure of the Ebre, Llobregat and La Tordera river deltas (Bayó y Custodio, 1989).

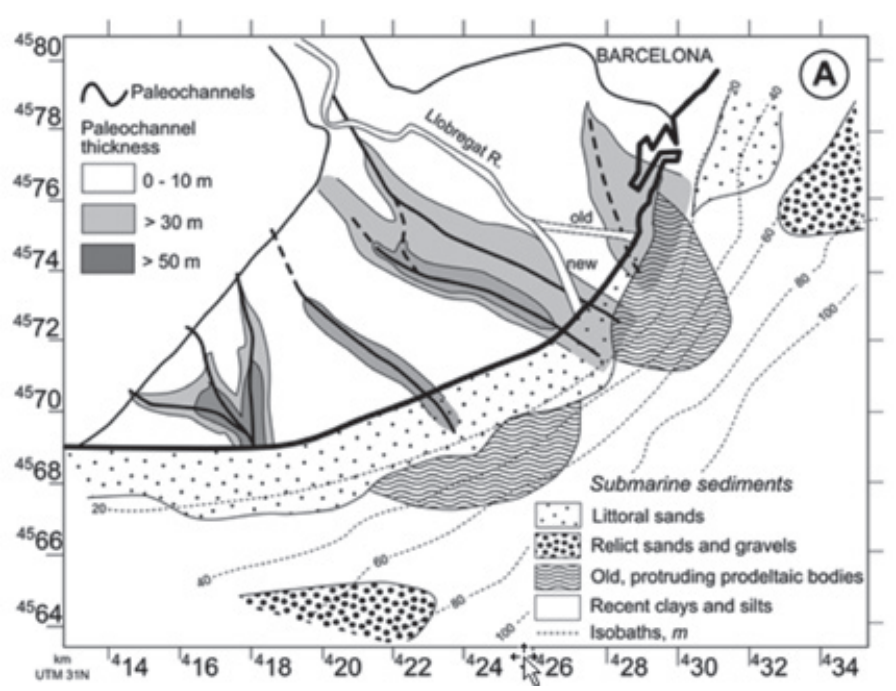

Figura 7. Heterogeneidad del acuífero profundo del delta del Llobregat, modificado de Serra Raventós y Verdaguer Andreu (1983), extendido y tomado de Simó et al. (2005). Además de los depósitos aluviales en el entorno del valle principal, cuyo delta se encontraría mar adentro respecto a la costa actual, están los depósitos aluviales y torrenciales de los arroyos y barrancos de las áreas laterales. Quizás no haya depósitos aluviales entre ellos, aunque puede haber restos arenosos de barras litorales y dunas.

Figure 7. Heterogeneity of the Llobregat delta deep aquifer, modified from Serra Raventós and Verdaguer Andreu (1983), expanded and taken from Simó et al. (2005). Apart from the alluvial deposits near the main valley, where the delta should be offshore of the current shoreline, there are the alluvial and torrential deposits of the lateral areas. It is possible that there are no alluvial deposits, although some sandy remnants of littoral spits and dunes may be found. en el borde este del Campo de Cartagena (provincia de Murcia), donde la descarga incrementada de agua salobre del acuífero superficial, con alto contenido en nitratos, se combina con el aporte de fosfatos por los vertidos a las ramblas, dando lugar a un muy serio problema persistente de eutrofización de lo que antes había sido una gran laguna litoral de agua algo hipersalina, transparente y con notables valores ecológicos, turísticos y pesqueros;

3) Efecto de la explotación del agua subterránea en los acuíferos costeros, lo que causa la reducción y la posible desecación de humedales, cambios en la distribución de salinidad, tanto en horizontal como en vertical, y la disminución de la descarga de agua continental o insular al mar litoral, y;

4) Uso de agua salina subterránea para alimentar plantas de desalinización y desalobración. La extracción puede alterar las condiciones del agua en el litoral e interior como consecuencia del descenso de niveles piezométricos y la infiltración de las salmueras residuales del tratamiento, si no se ha adoptado una solución adecuada, que es costosa y a veces complicada. En el caso de áreas agrícolas abastecidas con desalobradoras alimentadas con aguas freáticas locales, las salmueras de retorno (rechazo) pueden tener altos contenidos de nitratos, además de otros solutos, que pueden afectar a las condiciones ecológicas en las áreas de descarga. Tal es el caso del Mar Menor, antes mencionado.

5) Acumulación en el área costera de aguas subterráneas captadas y en parte vertidas (por ejemplo, retornos de riego) o de fuga de conducciones, creando encharcamientos más o menos permanentes que limitan y dificultan el uso del territorio. Tal sucede en la Balsa del Sapo, en el Campo de Dalías.

La apreciación y cuantificación de los efectos de la explotación sobre la salinización y la ecología puede ser complicada cuando se trata de estructuras geológicas complejas, como es el caso del Campo de Cartagena, en las que existen diferentes acuíferos superpuestos, con explotación que puede afectar a varios de ellos a la vez, pero con contacto directo con el mar limitado a las formaciones más superficiales. A esta situación en relación directa con la hidrogeología hay que añadir los condicionantes hidrodinámicos, de recarga y en su caso de posibles aguas salinas de otro origen que el marino actual, como los retornos de riego, las aguas marinas residuales antiguas o la disolución de minerales evaporíticos (Jiménez-Martínez et al., 2011; 2012).

Apenas hay estudios detallados de cómo la descarga de agua subterránea en el litoral afecta a los ecosistemas y a la pesca. García-Solsona et al. (2010) han realizado intentos asociados a las descargas por los carbonatos de la Serra d'Irta (provincia de Castelló). 


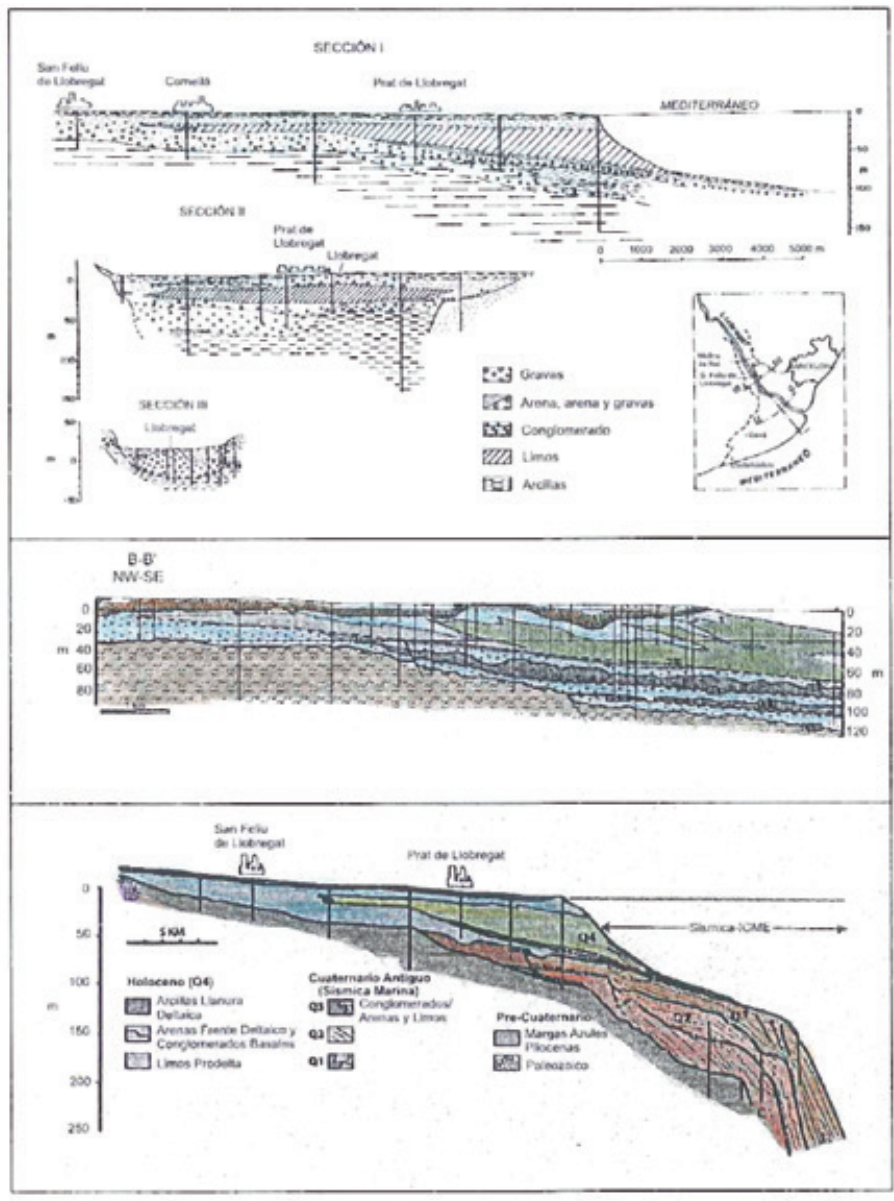

Figura 8. Cortes geológico-sedimentológicos del delta del Llobregat según el trazado del río, en el propio delta (figura superior) y comprendiendo el valle bajo y la extensión submarina (figura inferior), según Gámez (2007).

Figure 8. Geological-sedimentological cross-sections of the Llobregat delta following the river, in the delta itself (upper figure) and comprising the lower valley and the offshore extension (lower figure), Gámez (2007).

No se considera en modo alguno, de forma explícita, el efecto sobre las aguas litorales de la descarga de agua subterránea continental. El plan hidrológico de la Demarcación hidrográfica del Júcar (Xúquer) considera y estima el caudal de agua subterránea que se debe dejar descargar al mar, para prevenir o restaurar la distribución de salinidad en el acuífero y en determinados humedales costeros, pero sin aludir a la ecología litoral. Esto se detalla algo más para los acuíferos de la Plana de Castelló (IGME-UJI, 2009), pero desde el punto de vista de limitar la intrusión marina. En el plan hidrológico del Distrito fluvial de Cataluña se hace algo similar, aunque con menos detalle. De acuerdo con la normativa española de planificación hidrológica, los caudales determinados se restan de los recursos de agua subterránea disponible antes de definirlos y atribuirlos a los distintos usos.

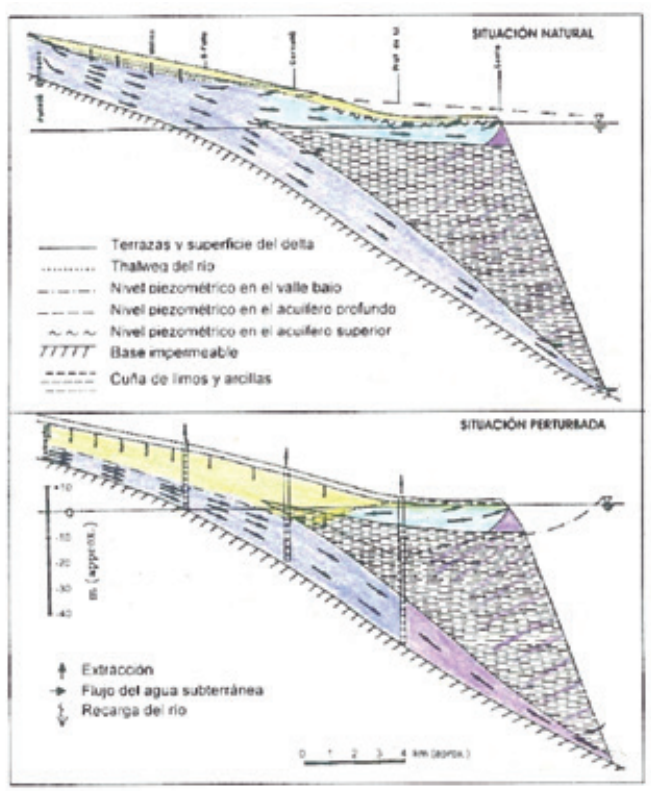

Figure 9. Esquema longitudinal del sistema acuífero bicapa del delta del Llobregat y su conexión con el valle bajo. Se muestra la situación natural previa a la explotación intensiva y la que corresponde al periodo de máxima extracción y casi nueva estabilización (Custodio, 2008; 2012).

Figure 9. Longitudinal diagram of the two-layer Llobregat delta aquifer system and its connection with the low valley. The natural predevelopment situation is shown and that in the period of maximum abstraction and the almost new stable situation (Custodio 2008; 2012).

\section{Restricciones administrativas y legales}

La legislación de agua vigente en España no considera directamente a los acuíferos costeros, ya que estos son un asunto específico y de detalle. La normativa aplicable es indirecta y se tiene que derivar de las normas que hacen referencia a la calidad del agua y a la protección de humedales.

Antes de 1985, el agua subterránea era del dominio privado y por lo tanto la administración pública del agua no se interesaba por ella sino en lo que podía afectar a las aguas públicas. El conocimiento y marco conceptual eran muy limitados, por el escaso conocimiento de los procesos hidrológicos. Hubo algunas excepciones en casos de gran competencia por el agua y alta litigiosidad entre los explotadores. Tal es el caso de las Islas Canarias desde 1924, luego reconfirmado en 1959, y de las Islas Baleares desde 1970. El efecto práctico sobre los acuíferos costeros fue pequeño, salvo excepciones, como en el caso del acuífero deTelde-Vecindario, en el E de Gran Canaria.

Según la Ley de Aguas de 1985 (LA, 1985), luego modificada en el Texto Refundido de la Ley de Aguas 


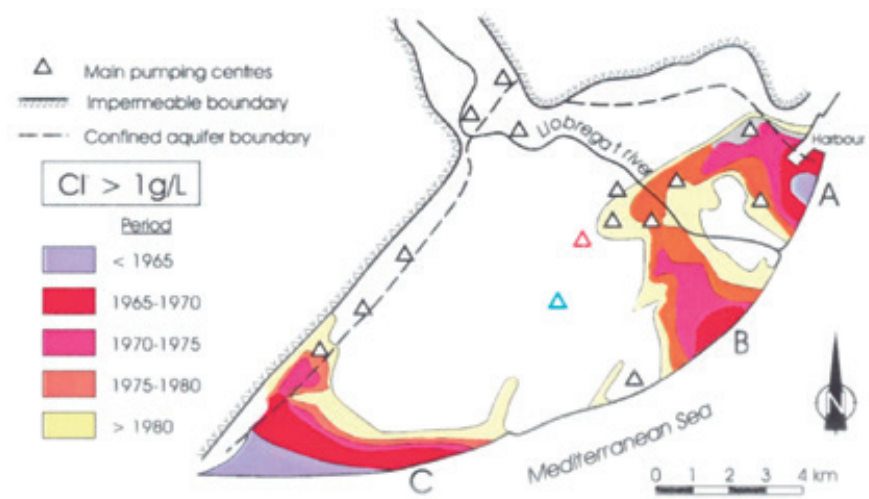

Figura 10. Evolución del contenido en cloruros (>1 g/L) del agua subterránea del acuífero profundo del delta del Llobregat entre 1965 y 1985 (Iribar y Custodio, 1992; Custodio, 2012).

Figure 10. Evolution of the chloride content ( $>1 \mathrm{~g} / \mathrm{L}$ ) in groundwater from the deep aquifer of the Llobregat delta, between 1965 and 1985 (Iribar and Custodio, 1992; Custodio, 2012).

de 2003 (TRLA, 2001; 2003) y cambios posteriores, todas las aguas son del dominio público. No obstante, los propietarios de pozos con titularidad anterior a 1985 , cuando se trataba de un dominio privado, pueden conservar sus derechos si lo desean, opción a la que expresa o tácitamente se han adherido gran parte de los explotadores de agua subterránea preexistentes. La atribución de responsabilidades de administración y gestión sobre las aguas subterráneas a las autoridades públicas del agua (Confederaciones y Servicios Hidráulicos insulares) en 1985 supuso un drástico cambio y ampliación para las tareas para las que estaban diseñadas y entrenadas, para las que además no tenían personal formado en hidrogeología, y que en parte siguen sin tener un buen número de ellas. En la realidad, el esfuerzo de estudio y observación decayó y no se ha recuperado en términos relativos si se conidera que los problemas han aumentado y que hay notables nuevas exigencias tras la incorporación de España a la Unión Europea.

La transposición de la Directiva Marco del Agua europea del año 2000 (DMA, 2000) ha trasladado el interés hacia el buen estado de las aguas continentales y su calidad. Esto está relacionado indirectamente con la salinización de los acuíferos costeros. La administración pública del agua española ha puesto su interés preferente en la caracterización del estado cuantitativo y cualitativo de las aguas subterráneas, con medios limitados y no siempre con el debido soporte científico, pero no en las causas y soluciones a los problemas de salinización mediante gestión del acuífero. Los esfuerzos se han dirigido en numerosos casos a abandonar el uso del acuífero o a su recuperación temporal y a substituir los recursos de agua perdidos por impor-

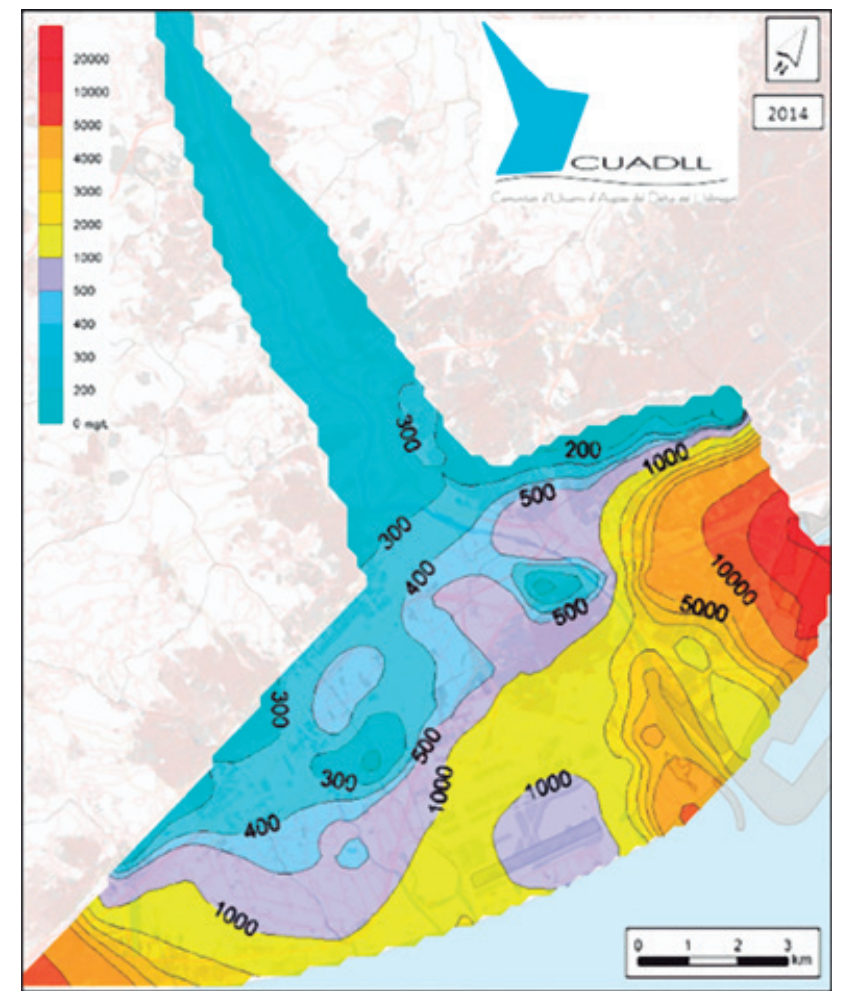

Figura 11. Contenido en cloruros $(\mathrm{mg} / \mathrm{L})$ del acuífero profundo del delta del Llobregat en 2014, según la CUADLL (Comunitat d'Usuaris d'Aigües del Baix Llobregat).

Figure 11. Chloride content ( $\mathrm{mg} / \mathrm{L}$ ) in the deep Llobregat delta aquifer in 2014, from CUADLL (Water Users' Community of the Lower Llobregat).

taciones, nuevas obras en la cuenca, regeneración de aguas urbanas usadas o desalinización. Estos recursos de agua son frecuentemente más caros y conllevan actuaciones ecológicamente más agresivas.

Con fines administrativos y de gestión, cabe utilizar preliminarmente indicadores relativos al estado de la intrusión marina, como comentan Werner et al. (2012), y valoraciones de la vulnerabilidad correspondiente (Azizi et al., 2019). Sin embargo, esta es una tarea difícil - quizás imposible-ya que intervienen muy diversas variables que tienen notable peso y que con frecuencia son de valor desconocido si no hay estudios y observaciones con un mínimo de detalle. La Confederación Hidrográfica del Júcar y el IGME han realizado esfuerzos en este sentido (Ferrer et al., 2007; Gómez et al., 2012), pero es sólo una preliminar aproximación.

\section{Efectos económicos y sociales}

La salinización de acuíferos costeros comporta pérdidas económicas. Parece que no han sido cuantificadas hasta ahora. Estas pérdidas son principalmente las asociadas a tener que aportar agua dulce de otros 


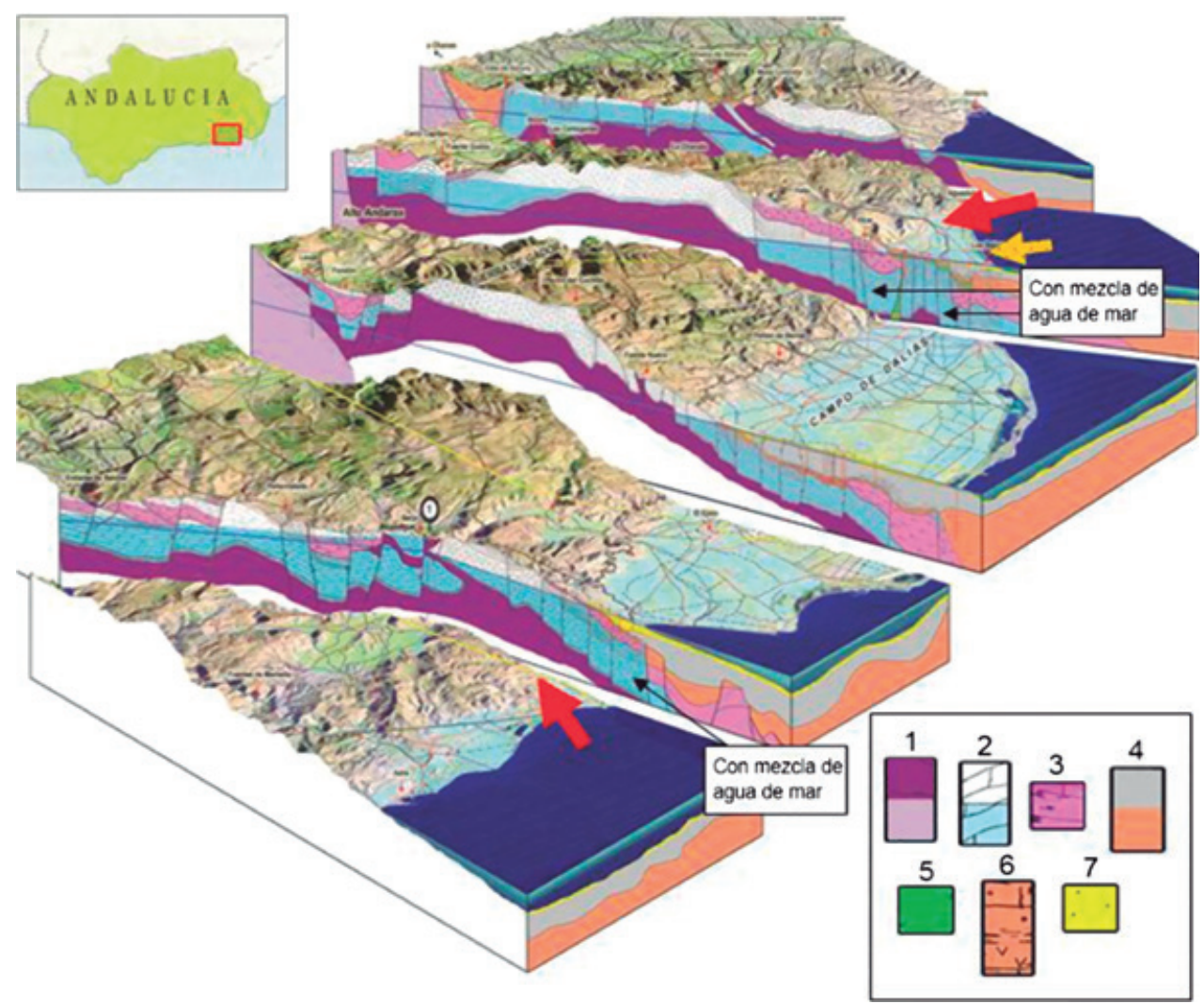

Figura 12. Estructura del Campo de Dalías-Sierra de Gádor mostrada mediante bloques diagrama (Domínguez et al., 2015; Domínguez Prats, 2017). 1, 3, 4 y 5 son formaciones impermeables; 6 y 7 son acuíferos neógenos de cobertera; 2 son acuíferos inferiores en carbonatos triásicos. La zona de recarga está en blanco y la de acumulación de reservas de agua subterránea en azul.

Figure 12. Structure of the Campo de Dalías-Sierra de Gádor shown by means of block-diagrams (Domínguez et al., 2015; Domínguez Prats, 2017). 1, 3, 4 and 5 are impermeable formations; 6 and 7 are covered Neogene aquifers; 2 are deep aquifers in Triassic carbonates. The recharge zone is in white and that of the groundwater reserve accumulation in blue.

orígenes, el mayor coste de los dispositivos, estructuras y redes de distribución domésticas, urbanas e industriales a causa de la mayor corrosión y el incremento de fugas asociado y la menor productividad agrícola, con modificación de cultivos, abandono de parcelas y necesidad a veces de realizar mezclas de agua en dispositivos apropiados. Este mayor coste se paga comúnmente por los usuarios del agua, muchos de los cuales no participan de los beneficios de la extracción de agua subterránea. Un coste adicional es el de tener que abandonar o substituir prematuramente los dispositivos de extracción y manejo del agua. Además, se tiene el coste asociado al deterioro de los servicios ecológicos de los humedales costeros, que actualmente es mal conocido y se tiende a olvidar. En muchos lugares donde el agua subterránea es salobre han proliferado las plantas de reducción de la salinidad mediante ósmosis inversa, a veces de electrodiálisis reversible, principalmente para riego de cultivos de alto precio en el mercado y en instalaciones turísticas. Esto es un nuevo coste adicional directo. En el
Campo de Cartagena (provincia de Murcia) hay numerosas pequeñas plantas de este tipo, posiblemente más de 1000 (Aparicio et al., 2017). Las externalidades negativas asociadas al vertido de la salmuera de rechazo es otro coste adicional importante, aunque es frecuente que no se tenga en cuenta y ello explica parte de los problemas que aparecen en diversas áreas, tales como el Campo de Cartagena (Región de Murcia) y el Campo de Níjar (provincia de Almería).

En algunos casos, los grandes descensos del nivel freático que se produjeron en áreas urbanas e industriales costeras permitieron y permiten usar el espacio no saturado subterráneo para ubicar instalaciones, almacenes y transporte, con obras sencillas y sin problemas de agua. El cese de las extracciones de agua subterránea en el área a causa de la salinización supone un ascenso freático y la consiguiente inundación de los espacios subterráneos habilitados o aparición de zonas encharcadas que excluyen otros usos urbanos o agrícolas. Esto supone un coste de drenaje por bombeo, con el consiguiente aumento de la salinidad 


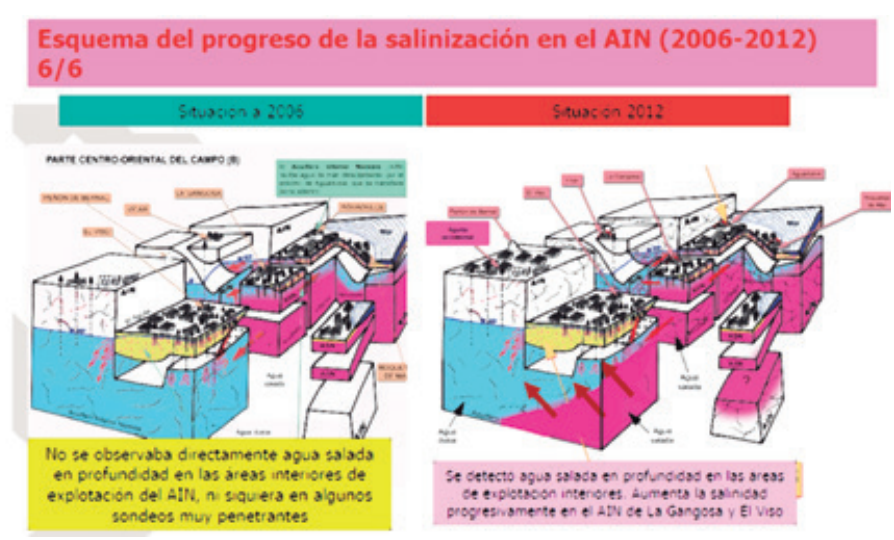

Figura 13. Explicación mediante diagramas-bloque del proceso de intrusión marina en los acuíferos profundos del Campo de Dalías (acuífero inferior norte), comparando la situación en 2006 con la de 2012 (Domínguez et al., 2013).

Figure 13. Explanation by means of block-diagrams of the seawater intrusion in the deep aquifers of the Campo de Dalías (lower deep aquifer), comparing the situation in 2006 with that in 2012 (Domínguez et al., 2013).

del agua extraída por los vecinos y la posible salinización de las aguas del alcantarillado si se vierte en el mismo, lo que impide la reutilización. El vertido sin problemas del agua de drenaje es un nuevo y en general importante coste a considerar. También hay que considerar el coste del abandono de inversiones e infraestructuras antes de su amortización prevista.

La posible subsidencia asociada a la extracción de agua subterránea en sedimentos costeros en proceso de compactación es otro aspecto a considerar (Pulido-Bosch et al., 2012), que conlleva costes económicos y problemas territoriales y sociales. En el área costera mediterránea e insular española apenas se han detectado problemas singulares de este tipo, posiblemente por falta de estudios al respecto. En el delta del Llobregat, parte del retroceso de la línea de costa es explicable como el resultado combinado de la compactación natural con la que se ha producido en el acuitardo intermedio y depósitos deltaicos superiores (estimado en unos 0,5 m) a causa de los grandes descensos piezométricos en el acuífero profundo por las extracciones de aguas subterráneas, el lento aumento del nivel del mary la disminución del aporte de sedimentos al mar.

Un notable efecto social, con serias repercusiones económicas e incidencia sanitaria, se produce cuando la explotación de un acuífero costero hace que las captaciones se salinicen y no se dispone de otra fuente de agua. Fue un serio problema en el área de Tarragona entre 1995 y 2005 , en los que el agua suministrada sólo se podía utilizar para usos sanitarios, y eso con corta vida de las instalaciones y aparatos do- mésticos e industriales. Pero esa situación, con menor intensidad, también existió en muy diversos lugares del litoral español continental e insular. Se ha paliado con importación de agua de otras áreas o desalinización, pero persisten situaciones de gravedad en Mallorca, Eivissa y Telde-Vecindario (Gran Canaria). La aplicación de la normativa europea ha obligado a gestionar los problemas que anteriormente no se habían abordado por iniciativa de las autoridades del agua.

\section{Actuaciones de gestión}

Las actuaciones de gestión pública para resolver problemas de salinización en acuíferos costeros han sido y son dominantemente estructurales. Pocas veces se han considerado las acciones no estructurales, que requieren que la sociedad civil se involucre y coopere, planes bien diseñados, personal especialmente entrenado y un largo tiempo para dejar que aparezcan los frutos y estos se puedan comunicar a los ciudadanos. Esto difícilmente cuadra con los tiempos de la política, a menos de que exista una presión estable y efectiva por parte de la sociedad civil. Entre las acciones no estructurales, la del establecimiento de tasas específicas no parece haber funcionado bien en otros paises $y$ suele inducir serios actos de oposición.

La mayoría de actuaciones estructurales han sido para poner a disposición nuevas fuentes de agua. A gran escala, en el sudeste español se trata del transvase de agua desde las cuencas atlánticas mediante los acueductos Tajo-Segura y Negratín-Almanzora. Además, se han distribuido a lo largo de la costa unas 20 grandes plantas de desalinización del agua del mar (Baltanás, 2006; Cabrera et al., 2019; Downward y Taylor, 2007) y se riega con agua residual urbana tratada hasta lograr los estándares establecidos para ese uso. Esta reutilización ha sido un gran éxito en la Cuenca del Segura, que ha merecido reconocimiento internacional.

La puesta a disposición de agua adicional supone en general un notable incremento del coste del agua, en especial cuando se trata de agua de mar desalinizada. Para promover que se utilicen los nuevos recursos de agua, en especial los de desalinización, en algunas áreas se han establecido subsidios directos o indirectos, incluso en algunos casos con tarifas que no cubren los costes de operación. Esto distorsiona la economía regional.

En el Área Metropolitana de Barcelona, la situación es diferente ya que se trata de un sistema integrado de recursos de agua, que combina agua fluvial propia con agua transvasada, explotación de los acuíferos locales como fuente y como reserva de sequía, recarga artificial de los acuíferos, reutilización de aguas urbanas resi- 
duales tratadas y desalinización. Se busca garantizar la disponibilidad de agua urbana en cantidad y en calidad, al menor coste y con la mayor seguridad, teniendo en cuenta las frecuentes situaciones de sequía. En épocas en que se debe extraer agua durante un largo tiempo de los acuíferos locales, en especial del Baix Llobregat, que incluye al delta, se produce un incremento de la intrusión marina. Es algo aceptable hasta una determinada situación, que se modela numéricamente. Para limitar dicha intrusión y para reducir la preexistente, se dispone de una barrera de 13 pozos costeros de inyección al acuífero profundo, según una línea paralela a la costa. Se opera cuando es necesario. El agua de inyección es agua residual urbana, que tras el tratamiento terciario recibe un afino adicional que incluye microfiltración, desinfección con rayos ultravioleta y ósmosis inversa (Ortuño et al., 2012a; 2012b). Las Figuras 14 y 15 muestran la ubicación de la barrera hidráulica de inyección y el efecto de la primera fase de inyección con sólo el primer tercio de la barrera en operación.

Los acuíferos costeros con agua salobre pueden ser una fuente de agua dulce tras un tratamiento de desalobración, como se ha comentado anteriormente.También de los mismos se puede captar agua de salinidad similar a la marina para alimentar plantas de desalinización, exenta de turbidez y componentes biológicos. Se tiene una buena experiencia en España (RodríguezEstrella y Pulido-Bosch, 2009). Pero hay aspectos negativos, además de las dificultades para obtener de los acuíferos costeros los altos caudales de agua cruda requerida, como el hecho de lo que se bombea - salvo en situaciones especiales - es agua marina con cierto tiempo de tránsito por el acuífero, con la adición de un cierto caudal de agua dulce continental o insular. Esto no sólo supone una modificación química que afecta al modo de operación de la planta, sino una pérdida de un recurso de agua dulce de bajo coste - que hay que bombear $-y$ problemas con los otros explotadores del acuífero (Cabrera et al., 2012) y en general con los habitantes en áreas escasas de agua. Hay algunos fracasos de esta forma de captación, mayormente a causa de estudios hidrogeológicos previos insuficientes, como en la planta de LaTordera (provincia de Girona). Esta forma de abastecimiento ha sido cuidadosamente estudiada para la planta desalinizadora de Almería, en el Bajo Andarax (Pulido-Bosch et al., 2004; 2019; Pulido-Bosch y Rodríguez Estrella, 2007).

\section{Consideraciones sociales}

El aumento de la intrusión marina supone una pérdida de recursos de agua dulce, tanto por reducción del almacenamiento de agua dulce como por salini- zación. Esto supone afecciones sociales, que incluyen las económicas y las ambientales. En cada caso son distintas y con diferente gravedad, en función de la escasez de agua dulce y de las variaciones estacionales e inter-anuales de la disponibilidad. En la Tabla 2 se da un resumen general de las consecuencias de la explotación de los acuíferos costeros, que debe adaptarse a las circunstancias reales de cada caso.

La buena gobernanza de los acuíferos costeros, como parte de la buena gobernanza del agua, es un asunto que ha sido poco tenido en cuenta, aunque se avanza en su consideración. La Ley de Aguas de 1985 consideraba la existencia de acuíferos denominados "sobrexplotados" en los que se debía aplicar medidas para corregir la situación. No hay referencia expresa a la intrusión marina, pero esta puede considerarse como un aspecto de esa "sobrexplotación". En la reforma de la Ley de Aguas (TRLA, 2001: 2003) para transponer la DMA (2000), este concepto de "sobreexplotación" se esconde tras el de buen estado de las aguas subterráneas, pero subsiste en la planificación hidrológica española. Esto supone actuaciones de arriba abajo (de la autoridad hacia el usuario) para constituir comunidades de usuarios que apliquen las normas de gestión establecidas por las autoridades del agua a través de los planes hidrológicos. El resultado real ha sido negativo y no se han constituido, salvo donde se han acordado notables inversiones públicas en obras y subvenciones para su logro, como el Medio y Alto Vinalopó, aunque en este caso sólo afecta marginalmente a acuíferos costeros.

En lugares con serios problemas conocidos y sentidos por los usuarios se han formado tales asociaciones en un proceso de abajo arriba. Han resultado positivas, aunque con logros reales variables en cada caso. Se trata de una acción pionera a nivel mundial, ya que las existentes en México, India y China tienen una notable incidencia de la promoción oficial y en California la legislación propia configura otro tipo de instituciones. En la legislación española de aguas, estas asociaciones, tanto unas como otras, denominadas Comunidades de Usuarios de Aguas Subterráneas (CUAS), son de derecho público y gozan de sus ventajas (Codina, 2004).

La primera asociación de usuarios de aguas subterráneas nació en 1975 en el Baix Llobregat. Es un acuífero costero ya que comprende el delta. La CUADLL (Comunitat d'Usuaris d'Aigües del Baix Llobregat) se formó con anterioridad a la Ley de Aguas de 1985, cuando las aguas subterráneas eran del dominio privado. Está constituida principalmente por abastecedores de agua urbana e industrias, aunque los regantes también forman parte (Queralt i Creus, 2007). El sector turístico está mayormente conectado a redes públicas 
de abastecimiento. Actualmente la tutela corresponde a la Agéncia Catalana de l'Aigua.

En España hay actualmente 20 CUAS de promoción de los usuarios, de las que 8 son costeras o tienen una parte costera (Rica Izquierdo, 2016; SASMIE, 2017): 1976 Baix Llobregat, 1986 PDO Campo de Dalías y Bajo Andarax, 1991 Junta Central de Usuarios del Poniente Almeriense, 1998 Agrupación Provincial de Pozos de Riego de Castellón, 1999 Agrupación de Pozos de Riego de la Comunidad Valenciana, 2000 Junta Central de Usuarios de los Acuíferos de la Sierra de Gádor, 2010 Junta Central de Usuarios del MedioBajo Andarax y 2016 Baix Empordà. El año indicado es el de constitución oficial.

No se han constituido CUAS en las Islas Baleares. Los intentos para constituirlas en Canarias no han progresado. En parte se explica por la existencia previa de Heredades y Comunidades de Agua y grandes explotadores y de mercados de aguas en Gran Canaria y Tenerife, lo que establece relaciones entre los principales consumidores y explotadores para defender, tratar y mejorar asuntos que les conciernen (MASE, 2015). Los respectivos Consejos Insulares han adoptado medidas, reflejadas en los decretos de los respectivos planes hidrológicos, para limitar los problemas de salinización costera de los acuíferos, como la clausura de pozos salinizados - en especial los que aportan a las redes de distribución generales - y la no autorización de nuevas captaciones, aunque en Gran Canaria subsiste la extracción de agua salobre para alimentar plantas desalobradoras. La Asociación Canaria de Empresarios del Agua, en Gran Canaria, actualmente es poco operativa. Con el apoyo del Consejo Insular de Aguas se han constituido sociedades y comunidades privadas para la gestión de aguas, para atender a la demanda agrícola y desalinizar, aunque también pueden dar servicio al abastecimiento urbano, pero no son CUAS. En Tenerife, la Cámara de Aguas tuvo un importante papel.

Es más fácil constituir CUAS en áreas en las que domina el abastecimiento y la industria, en parte por ser pocos los titulares de derechos para el inicio y en parte por el general mejor conocimiento del acuífero y de los riesgos de futuro (Rica et al., 2012; SASMIE, 2017). En el ámbito rural es más difícil la constitución ya que son muchos más los titulares, son más diversos los objetivos y sensibilidades, es más difícil que se reconozca que el acuífero es una herencia a infraestructura común suya que es necesaria para mantener las actividades económica y son menores los conocimientos. No obstante, este conocimiento puede ser notable allí donde los organismos públicos, principalmente el IGME, dedicó un prolongado y notable esfuerzo al conocimiento y observación. Otra dificul- tad añadida es la que se deriva de la coexistencia de agricultores regantes con y sin derechos de extracción de agua subterránea. Los primeros no comparten su ventaja relativa con los otros, ya en momentos de sequía, cuando otras fuentes de agua escasean o faltan, disponen de un recurso que puede ser objeto ventajoso de uso exclusivo o de comercio. Tal es el caso del Campo de Cartagena.

En la Comunidad Valenciana están muy implantadas las agrupaciones de gestión de aguas subterráneas, que en el caso de la agricultura son comunidades de regantes, algunas de ellas integradas en asociaciones de mayor entidad. Para la gestión del agua concedida, en 1989 se constituyó la Comunidad General de Regantes de La Vall d'Uixó, que es mixta de aguas superficiales y aguas subterráneas y corresponde a un acuífero costero.

La salinización e intrusión marina en acuíferos costeros tiene aspectos éticos y morales a considerar. La ética considera el comportamiento humano en relación con las normas de buena convivencia y respeto mutuo que tiene establecida la sociedad, con pautas comunes estables sobre lo que se considera bueno y honesto. La moral va más allá, al reconocer que el hombre es transcendente y debe respetar las leyes divinas, las cuales sirven de anclaje y son independientes de lo que es costumbre en la sociedad del momento (MASE, 2015; SASMIE, 2017).

Los acuíferos costeros de lenta renovación que se salinizan, degradan o son alterados por perforaciones inadecuadas, no van a estar disponibles para su uso futuro, al menos durante algunas generaciones humanas. Cabe considerar que lo que se ahorre actualmente para ser usado en el futuro puede producir beneficios a las generaciones venideras. Sin embargo, su evaluación es objeto de notables discrepancias en función de lo que se considere como valor de la tasa de descuento a aplicar, lo que es una decisión social sobre la que no hay acuerdo general El establecimiento del valor de dicha tasa tiene matices éticos.

El colapso o rápida degradación de las actividades sustentadas por un acuífero costero es no sólo una pérdida patrimonial y económica sino también social, lo que traslada responsabilidades éticas a los políticos, autoridades, usuarios y sociedad civil. En este sentido cabe encuadrar lo que se ha hecho para mantener operativo el acuífero del Baix Llobregat y su integración en el sistema de recursos hídricos y los serios problemas pasados del delta del Besós y de las zonas costeras del entorno de Tarragona y el Baix Camp de Tarragona y en el abastecimiento de Las Palmas de Gran Canaria por la intrusión marina en Telde. Actualmente los serios problemas de eutroficación del Mar Menor están en la misma línea, en este caso con la 


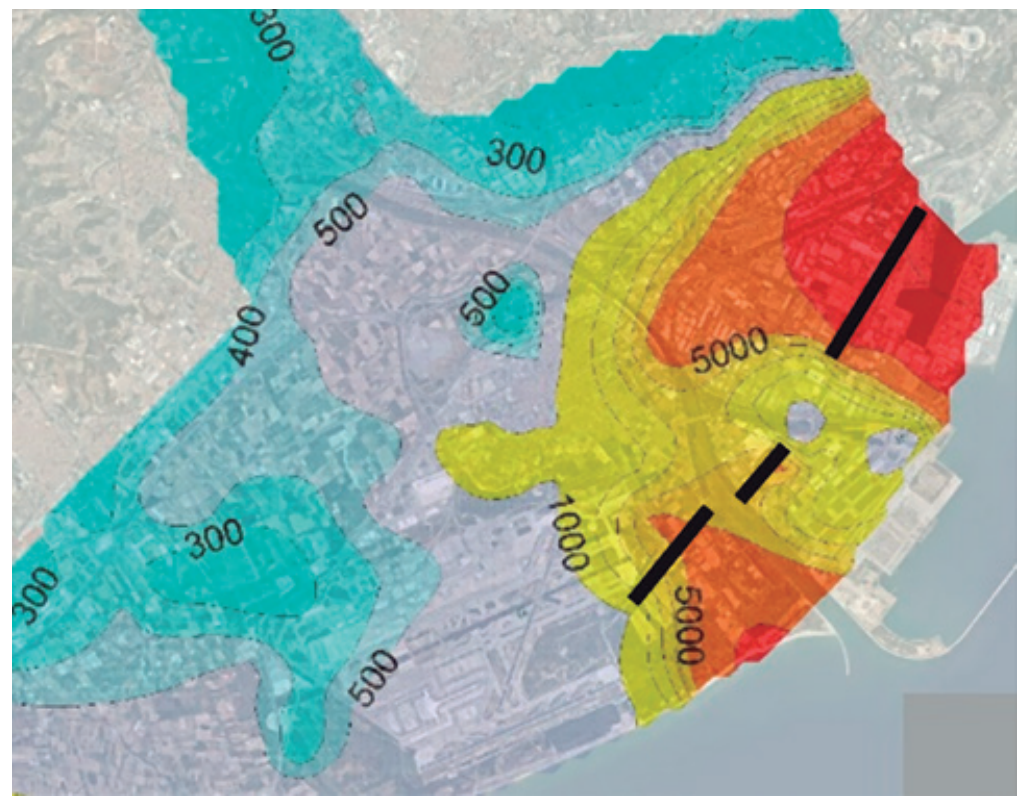

Figura 14. Ubicación de la barrera hidráulica de inyección en tramos preferenciales de intrusión marina en el acuífero profundo del delta del Llobregat. Se muestran las tres fases de construcción, siendo la primera la central. Los valores son el contenido en cloruros en mg/L Cl. Figure 14. Emplacement of the hydraulic injection barrier layout in preferential zones of marine intrusion into the deep Llobregat delta aquifer. The three phases of construction are indicated, the first the central one. The values are chloride concentration in groundwater, in $\mathrm{mg} / \mathrm{L} C \mathrm{Cl}$

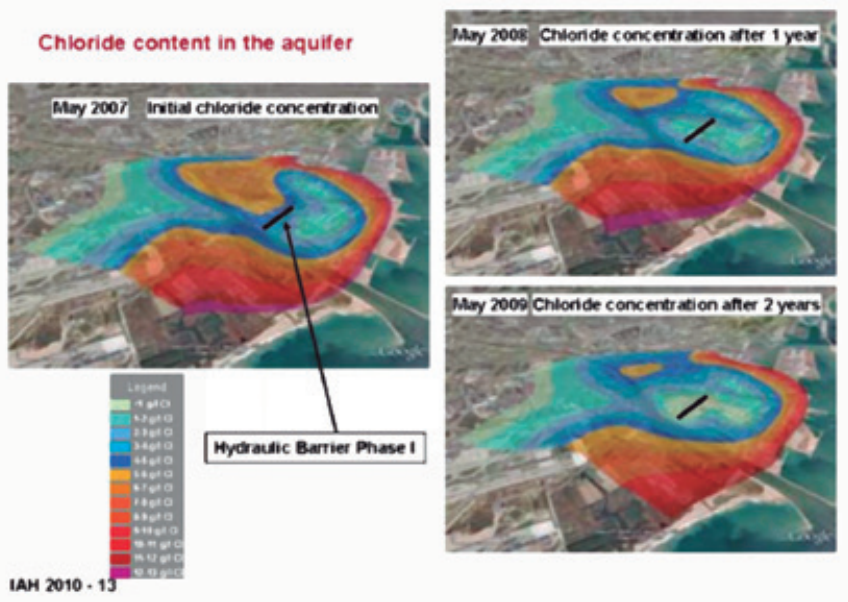

Figura 15. Efecto de la barrera positiva de inyección para el control de la intrusión marina en el acuífero profundo del delta del Llobregat tras la actuación sólo en la parte central durante dos años (Ortuño et al., 2012a; 2012b). La escala de color es $<1 \mathrm{a}>12 \mathrm{~g} / \mathrm{L} \mathrm{Cl}$.

Figure 15. Effect of the positive injection barrier to control seawater intrusion in the Llobregat delta aquifer after the operation of only the central part for two years (Ortuño et al., 2012a; 2012b). The scale of colours is $<1$ to $>12 \mathrm{~g} / \mathrm{L} \mathrm{Cl}$. seria degradación de un gran espacio acuático, con un largo camino para la recuperación. La actual gran riqueza asociada al Campo de Dalías plantea problemas éticos (Petit et al., 2017), en buena parte en relación con la intrusión marina, con un notable riesgo de quiebra si no se actúa decididamente.

\section{Papel futuro de los acuíferos costeros}

Los problemas de salinización e intrusión marina en los acuíferos costeros vividos en el pasado, aunque en ocasiones mal documentados y resultantes de una falta de gestión, han creado un sentimiento entre muchos de los gestores y autoridades del agua españoles de que los acuíferos costeros son inmanejables y demasiado complicados, de modo que es mejor no tenerlos en cuenta. La realidad puede ser bien diferente, puesto que pueden proporcionar cerca de los lugares de demanda de agua no sólo una fuente permanente de agua dulce sino también una reserva para las estaciones secas. En el caso de acuíferos extensos permite afrontar los periodos de sequía característicos del área mediterránea e insular. Así, la conservación de lo que se tiene y la recuperación de lo que se ha degradado es un objetivo a considerar, siempre y cuando el coste de hacerlo no sea desproporcionado. De hecho, en el área costera de Barcelona se han restaurado y puesto en operación antiguos pozos de abastecimiento, para usos diversos, aunque para el uso potable se requiere 
adicionar algunos tratamientos costosos para conseguir y garantizar la calidad necesaria.

El papel de los acuíferos costeros se incrementará si las otras fuentes de agua merman, bien sea por cambios globales (expansión urbana, mayor población, reducción de las áreas de recarga) o por una posible evolución climática que lleve a una disminución de la recarga $(I A H, 2012)$ y de otros recursos de agua. Tal suele ser el actual pronóstico para el área mediterránea. En cualquier caso, el conocimiento de la recarga natural a los acuíferos costeros en el contexto de cada momento es importante para una adecuada gestión, a pesar de las dificultades e incertidumbres que entraña, como se desarrolla detalladamente en REAMIA (2019) y las numerosas referencias contenidas en ese trabajo. Entre los trabajos de gestión está el incremento de la recarga inducida y artificial para mantener la descarga de agua continental al mar, que ya se viene practicando en el Baix Llobregat desde 1949 (Custodio, 1986; Queralt et al., 2020). La barrera de control de la intrusión marina del delta del Llobregat antes presentada es uno de los elementos de recarga artificial.

En cualquier caso, se va a requerir cada vez mayor gestión integrada de los recursos de agua para afrontar la escasez relativa. Los acuíferos pueden aportar la capacidad de regulación que se necesita. Sin embargo, el uso de los acuíferos como reguladores necesita herramientas legales y administrativas, que sea posible tratar al acuífero como una unidad, a pesar de que en el mismo existan diversos propietarios y derechos, y que los usuarios de agua y la sociedad civil se involucren. Esto es algo que no está debidamente considerado en la Directiva Marco del Agua europea, en parte por ser una directiva orientada al buen estado del medio ambiente. Pero es difícil separar buen estado ecológico de buena gestión. Por esta razón, este es uno de los aspectos a considerar en las modificaciones que hay que introducir en la DMA al final de su periodo legal de vigencia en 2027, y que se empieza a discutir a partir de finales de 2019. Este aspecto es uno de los que se consideran en el informe para la Comisión Europea que han preparado a finales de 2019 la Real Academia de Ciencias de España y la Sociedad General de Aguas de Barcelona-SUEZ-España, que no se ha publicado. Cabe considerar que buen estado del agua no es posiblemente el mejor objetivo a conseguir si se mantienen las actuales definiciones, en especial en acuíferos costeros. Puede ser más adecuado buscar un buen estado razonable socialmente, que en el área semiárida mediterránea permita incluir el uso de los acuíferos como infraestructuras naturales (Custodio, 2005) y que los acuíferos (o las masas de agua subterránea) puedan tener vocaciones y consideraciones medioambientales distintas para diferentes usos.

\section{Agradecimientos}

El informe que soporta este artículo (SASMIE, 2017) ha sido realizado con el apoyo de SUEZ-España y CETAQUA. Se agradece el soporte personal de Manuel Cermerón, Carlos Montero, Xavier Bernat y Juan Antonio Barberá. Se agradece también el apoyo de numerosos expertos que han aportado información, experiencia y observaciones, que se han integrado en dicho informe y cuya relación figura en el mismo.

Positivas ミ beneficios
Abastecer agua
Desarrollo económico y social
Dar empleo
Fijar población en el territorio
Drenaje de terrenos

Tabla 2. Consecuencias de la explotación de los acuíferos costeros. Table 2. Consequences of coastal aquifer exploitation. 


\section{Referencias}

Aparicio, J.O, Candela, L., Alfranca, O. and García-Aróstegui, J. (2017). Economic evaluation of small desalination plants from brackish aquifers. Application to Campo de Cartagena (SE Spain). Desalination, 411: 38-44.

Azizi, F., Vadiati, M., Moghaddam, A.A., Nazemi, A. and Adamowski, J. (2019). A hydrogeological-based multi-criteria method for assessing the vulnerability of coastal aquifers to saltwater intrusion. Environmental. Earth Science,s 78:548-.

Baltanás, A. (2006). Spanish push for desalination: part of a larger plan. Desalination, 99: 57-71.

Bayó, A. et Custodio, E. (1989). Deltas et plaines côtières longitudinales de Catalogne. Notice explicative de la Feuille B6Madrid de la Carte Hydrogéologique Internationale de l'Europe 1/1500 000. UNESCO-BGR, Paris-Hannover: 69-76.

Bayó, A., Loaso, C., Aragonés, J.M. and Custodio, E. (1992). Marine intrusion and brackish water in coastal aquifers of Southern Catalonia and Castelló (Spain): a brief survey of actual problems and circumstances. In: Study and Modelling of Saltwater Intrusion into Aquifers. 12th Salt Water Intrusion Meeting. Barcelona: 741-766.

Bayó, A., Custodio, E. y Loaso, C. (1997). Las aguas subterráneas en el Delta del Ebro. Revista de Obras Públicas. Madrid. 3368: 47-65.

Bear, J. (2007). Seawater intrusion in coastal aquifers. In: A. Pulido Bosch, J.A. López-Geta y G. Ramos González, Los Acuíferos Costeros: Retos y Soluciones. III Simposio sobre Tecnología de la Intrusión Marina, Almería. Publicaciones del IGME, Serie Hidrogeología y Aguas Subterráneas 23, Madrid: I: 3-22.

Cabrera, E., Estrela, T. and Lora, J. (2019). Desalination in Spain. Past, present and future. Ingeniería del Agua, 23(3), 199-214. https://doi.org/10.4995/la.2019.11597

Cabrera, M.C. and Custodio, E. (2004). Groundwater flow in a volcanic-sedimentary coastal aquifer: Telde area, Gran Canaria, Canary Islands, Spain. Hydrogeology Journal, 12(3): 305-320.

Cabrera, M.C. and Custodio, E. (2005). Evolution of groundwater intensive development in the coastal aquifer of Telde (Gran Canaria, Canarian Archipelago, Spain). In: A. Sahuquillo, J. Capilla, L. Martínez-Cortina, X. SánchezVila (eds.), Groundwater Intensive Use. International Association of Hydrogeologists, Selected Papers 7, Balkema: 295-306.

Cabrera, M.C. y Custodio, E. (2012). Salinidad e intrusión marina en el acuífero de Telde (NE de Gran Canaria) y efecto de las plantas desaladoras. IV Simposio sobre Tecnología de la Intrusión Marina (TIAC), Alicante. I: 243-254.

Calvache, M.L. and Pulido-Bosch, A. (1997). Effects of geology and human activity on the dynamics of salt-water intrusion in three coastal aquifers in southern Spain. Environmental Geology, 30(3/4): 215-223.
Calvache, M.L.; Martín-Rosales, W.; Duque, C.; López-Chicano, M.; Rubio, J.C. y González-Ramón, A. (2007). Aguas subteráneas y acción antrópica en zonas costeras semiáridas: el acuífero Motril-Salobreña. III Simposio sobre la Tecnología de la Intrusión Marina (TIAC), Almería. II: 73-92.

Carrera, J.; Pool, M.; Abarca, E.; Hidalgo, J.J.; Slooten, L.J.; Vázquez-Suñé, E.; Sanz, E.; Gámez, D. y Alcolea, A. (2011). Principios y conceptos básicos sobre intrusión marina y gestión de acuíferos costeros. En: M.C. Cabrera, L.J. Lambán, M., Manzano, M. Valverde (eds.), Cuatro Décadas de Investigación y Formación en Aguas Subterráneas. Asociación Internacional de Hidrogeólogos-Grupo Español: 215-228.

Codina, J. (2004). Las aguas subterráneas: una visión social. El caso de la Comunidad de Usuarios del Llobregat. Real Acad. Cien. Exact. Fis. Nat. (Esp). Madrid, 98(2): 323-329.

Custodio, E. (1967). Études géohydrochimiques dans le delta du Llobregat, Barcelona (Espagne). International Association of Scientific Hydrology, Publ. 62: 134-155.

Custodio, E. (1976). Relaciones agua dulce-agua salada en las regiones costeras. En: E. Custodio \& M.R. Llamas (eds.). Hidrología Subterránea. Ediciones Omega. BarceIona, II-13: 1313-1389

Custodio, E. (1981a). Evaluación y causas de la contaminación por invasión de agua marina en los acuíferos de la costa peninsular y en las áreas insulares. En: Análisis y Evolución de la Contaminación de Aguas Subterráneas en España, Barcelona. AlH-GE. CIHS: 447-506.

Custodio, E. (1981b). Sea water encroachment in the Llobregat and Besós areas, near Barcelona (Catalonia, Spain). In: Intruded and Fosil Groundwater of Marine Origin. Salt Water Intrusion Meeting, Uppsala. Sveriges Geologiska Undersökning. Rapporter och Meddelanden 27: 120-152.

Custodio, E. 1986. Recarga artificial de acuíferos: avances y realizaciones. Boletín del Servicio Geológico-M.O.P.U. 45, España: 1-176.

Custodio, E. (1987). Sea-water intrusion in the Llobregat delta, near Barcelona (Catalonia, Spain). Groundwater Problems in Coastal Areas, UNESCO Studies and Reports in Hydrology 45, UNESCO, Paris: 436-463.

Custodio, E. (1990). Saline groundwater in the Canary Islands (Spain) resulting from aridity. In: Greenhouse Effect, Sea Level and Drought. NATO ASI Series C. Mathematical and Physical Sciences, 325. Reidel Publ. Co. Dordrecht: 593-618.

Custodio, E. (1992). Coastal aquifer salinization as a consequence of aridity: the case of Amurga phonolitic massif, Gran Canaria Island. 12th Salt Water Intrusion Meeting, Barcelona: 81-98.

Custodio, E. (2005). Coastal aquifers as important natural hydrogeological structures. In: E. Bocanegra, M. Hernández \& E. Usunoff, Groundwater and Human Development. Intenational Association of Hydrogeologists, Selected Papers 6. Balkema: 15-38. 
Custodio, E. (2008). Acuíferos detríticos costeros del litoral mediterráneo peninsular: valle bajo y delta del Llobregat. Monográfico: Las Aguas Subterráneas. Rev. Asoc. Española Enseñanza de las Ciencias de laTierra. Madrid, 15(3): 295-304.

Custodio, E. (2010). Coastal aquifers of Europe: an overview. Hydrogeology Journal, 18: 269-280.

Custodio, E. and Bruggeman, G.A. (1987). Groundwater problems in coastal areas. Studies and Reports in Hydrology, 45, UNESCO, Paris: 1-576.

Custodio, E. (2012). Low Llobregat aquifers: intensive development, salinization, contamination and management. In: S. Sabater, A. Ginebreda \& D. Barceló, The Story of a Polluted Mediterranean River. The Handbook of Environmental Chemistry, 21: 27-50.

DMA (2000). Directive 2000/60/EC of the European Parliament and of the 775 Council of 23 October 2000 establishing a framework for Community 777 action in the field of water policy. http://europa.eu.778int/eur-lex/pri/es/oj/ dat/2000/I_327/I_32720001222es00010072.pdf

Dominguez Prats, P. (2017). Aguas subterráneas e intrusión marina en el Campo de Dalías: cuatro décadas de estudios del IGME. En: SASMIE (2017), Salinización de las Aguas Subterráneas en los Acuíferos Costeros Mediterráneos e Insulares Españoles. Aportación específica 2, Sección 10.4.UPC e-books. http://hdl.handle.net/2117/111515

Domínguez, J.A., Franqueza, P.A., González, A. y Fernández, M.A. (2013). Acuíferos costeros del Campo de Dalías, datos del avance de su conocimiento por el IGME (destinado al asesoramiento) para un análisis científico sobre el interés y viabilidad de corregir sus tendencias negativas originadas por el uso. In: Antonio Fernández-Uria (ed.), X Simposio de Hidrogeología. Asociación Española de Hidrogeólogos-IGME, Granada. Hidrogeología y Recursos Hidráulicos XXX: 59-70.

Domínguez, J.A., Franqueza, P.A. y Fernández, M.A. (2015). Conocimiento de los acuíferos del sur de Sierra de Gádor-Campo de Dalías y su relación con la gestión. In: Situación Actual y Gestión de los Acuíferos del Sur de Sierra de Gádor-Campo de Dalías. Fundación Cajamar. Almería: 1-18.

http://www.fundacioncajamar.es/es/comun/transferencia/ jornadas-y-talleres/ano-2015/situacion-actual-y-gestionde-los-acuiferos-del-sur-de-la-sierra-de-gador-campode-dalias/

Downward, S.R. and Taylor, R. (2007). An assessment of Spain's "Programa AGUA" and its implications for sustainable water management in the province of Almería, Southeast Spain. Journal of Environmental Management, 82(2): 277-289.

Ferrer, J.; Fidalgo, A.; Estrela, T. y Esnaola, J.M. (2007). Metodología para la evaluación del riesgo por intrusión marina en las masas de agua subterránea costeras y resultados de su aplicación en la Confederación Hidrográfica del Júcar. II Simposio sobre la Tecnología de la Intrusión Marina (TIAC), Alicante, I: 751-760.

Gámez, D. (2007). Sequence stratigraphy as a tool for water resources management in alluvial coastal aquifers: application to the Llobregat delta (Barcelona, Spain). Department of Geotechnical Engineering and Geo-Sciences, Technical University of Catalonia (UPC), Barcelona. Doctoral Thesis: 1-177+An.

García-Arostegui, J.L., Jiménez-Martínez, J., Baudron, P., Martínez-Vicente, D., Senent, M. y Guerra, J. (2012). Geometría del acuífero del Campo de Cartagena e implicaciones en el funcionamiento hidrogeológico. IV Simposio sobre la Tecnología de la Intrusión Marina (TIAC), Alicante, I: 439-449.

García Aróstegui, J.L., Marín Arnaldos, F. y Martínez Vicente, D. (2017). Hidrogeología. En: Informe Integral sobre el Estado Ecológico del Mar Menor. Comité de Asesoramiento Científico del Mar Menor. Región de Murcia y Espacios Naturales. Cap. 1: 7-22.

http://canalmarmenor.es/download/40/comite-cientifico/1594/informe-integral-sobre-el-estado-ecologico-del-mar-menor.pdf

García-Menéndez, O., Morell, I., Ballesteros, B.J., Renau-Pruñonosa, A., Renau-Llorens, A. and Esteller, M.V. (2016). Spatial characterization of the seawater upconing process in a coastal Mediterranean aquifer (Plana de CasteIlón, Spain): evolution and controls. Environmental Earth Sciences, 75:728-.

García-Solsona, E., García-Orellana, J., Masqué, P., RodeIlas, V., Mejías, M., Ballesteros, B. and Domínguez, J.A. (2010). Groundwater and nutrient discharge through karstic coastal springs (Castelló, Spain). Biogeosciences, 7: 2625-2638.

Gómez Gómez, J.D., López Geta, J.A. and Garrido Schneider, E. (2003). The state of seawater intrusion in Spain. En: J.A. López Geta, J. de D. Gómez, J.A. de la Orden, G. Ramos y L. Rodríguez, Estado de la Intrusión de Agua del Mar en los Acuíferos Costeros del Mediterráneo y Técnica de Evaluación. II Simposio sobre Tecnología de la Intrusión Marina del Mar en Acuíferos Costeros: Países Mediterráneos, Alicante. Publicaciones del IGME, Serie Hidrogeología y Aguas Subterráneas, Madrid, II: 169-186.

Gómez, J.D., Ballesteros, B., Díaz, J.A., García, J.L., Haro, D., López, J.A., Meléndez, M., Pulido-Velázquez, D., Varela, M., Sánchez, I., Morell, A., Renau-Pruñonosa, A. y Mateu, J. (2012). Establecimiento de indicadores de intrusión marina y cálculo de descargas ambientales al mar en masa de agua subterránea costeras. In: L. Fernández, Las Aguas Subterráneas en la Planificación Hidrológica. IGME: 127-150.

Herrera, C. y Custodio, E. (2003). Hipótesis sobre el origen de la salinidad de las aguas subterráneas en la isla de Fuerteventura, Archipiélago de Canarias, España. Boletín Geológico Minero. 114(4): 433-452. 
$\mathrm{IAH}$ (2012). Climate changes effects on groundwater resources. A global synthesis of findings and recommendations. In: H. Treidel, J. L. Martin-Bordes \& J. Gurdak (eds.). Wallingford, UK. International Association of $\mathrm{Hy}$ drogeologists, Book Series: 1-398.

IGME (2000). Estado de la intrusión de agua de mar en los acuíferos costeros españoles. Volumen II. Cuencas mediterráneas I: Segura, Júcar y Baleares. Instituto Geológico y Minero de España. Madrid.

IGME-UJI (2009). Descargas ambientales al mar en las masas de agua subterránea costeras de la provincia de Castellón (Cuenca del Júcar). Instituto Geológico y Minero de España y Dirección General del Agua/Universitat Jaume I, Madrid/Castelló.

Iribar, V. and Custodio, E. (1992). Advancement of seawater intrusion in the Llobregat delta aquifer. 12th Salt Water Intrusion Meeting, Barcelona: 35-50.

Jiménez-Martínez, J., Aravena, R. and Candela, L. (2011). The role of leaky boreholes on the contamination of a regional confined aquifer: A case study in the Campo de Cartagena region, Spain. Water, Air \& Soil Pollution, 215: 311-327.

Jiménez-Martínez, J., Candela, L., García-Aróstegui, J.L. and Aragón, R. (2012). A 3D geological model of Campo de Cartagena, SE Spain: Hydrogeological implications. Geologica Acta, 10(2).

LA (1985). Ley de Aguas, Ley 28/1985, de 2 de agosto (BOE 189 de 8-8-1985: 25123-25135.

López-Geta, J.A. y Gómez-Gómez, J.D. (2007). La intrusión marina y su incidencia en los acuíferos españoles. Rev. Asoc. Enseñanza de las Ciencias de la Tierra, 153: 266-273.

López-Geta, J.A. y Fernández Ruiz, L. (2012). Importancia de los acuíferos costeros en España y el papel del TIAC. IV Simposio sobre Tecnología de la Intrusión Marina (TIAC), Alicante, II: 7-14.

Manzano, M., Custodio, E., Loosli, H., Cabrera, M.C., Riera, X. and Custodio-Ayala, J. (2001). Palaeowater in coastal aquifers of Spain. In: W.M. Edmunds \& C.J. Milne, Palaeowaters in Coastal Europe: Evolution of Groundwater since the Late Pleistocene. Geological Society (London), Special Publication 189: 107-138.

Margat, J. (2004). Atlas de l'eau dans le Bassin Méditerrranéen. UNESCO / Plan Bleu, Paris: 1-46.

Margat, J. and van der Gun, J. (2013). Groundwater around the World: A geographic synopsis. CRC Press: 1-376. ISBN 9781138000346 - CAT\# K2044.

MASE (2015). Aspectos hidrológicos, ambientales, económicos, sociales y éticos del consumo de reservas de agua subterránea en España: Minería del Agua Subterránea en España. Preparado por E. Custodio para UPC, Aqualogy y Cetaqua, Barcelona: 1-487. UPC e-books: http://hdl. handle.net/2117/111272.

MIMAM (2000). Libro blanco del agua en España. Ministerio de Medio Ambiente. Madrid
MOPTMA-MINER (1994). Libro blanco de las aguas subterráneas. Serie Monografías. Ministerio de Obras Públicas, Transporte y Medio Ambiente / Ministerio de Industria y Energía, Madrid: 1-135.

Ortuño, F.,Custodio, E., Molinero, J., Juárez, I., Garrido, T. and Fraile, J. (2012a). Seawater intrusion control by means of a reclaimed water injection barrier in the Llobregat delta, near Barcelona, Catalonia, Spain. In: P. Maloszewsky, S. Witczak \& G. Malina. Groundwater Quality Sustainability. International Association of Hydrogeologists, Selected Papers in Hydrogeology 17. CRC Press/Balkema: 199-210.

Ortuño, F., Molinero, J., Garrido, T. and Custodio, E. (2012b). Seawater injection barrier recharge with advanced reclaimed water at Llobregat delta aquifer (Spain). Water Science and Technology, 66 (10): 2083-2089.

Petit, O., Kuper, M., López-Gunn, E., Rimaudo, J.-D., Daudi, A. and Lejars, C. (2017). Can agricultural groundwater economics collapse? An inquiry into the pathways of four groundwater economies under threat. Hydrogeology Journal, 25(6): 1549-1564.

Pool, M., Abarca, E. y Carrera, J. (2007). Simplificaciones en la modelación de la intrusión marina: validez y alcance. Boletín Geológico y Minero, 118 (Especial): 593-608.

Post, V. y Abarca, E. (eds.) (2010). Saltwater and freshwater interactions in coastal aquifers. Journal of Hydrology, Special Issue 19(1): 1-270.

Pulido-Bosch, A., Pulido-Leboeuf, P., Sánchez-Martos, F., Gisbert, J. and Vallejos, A. (2002). Coastal aquifers and desalinisation plants. A case study: Almería, Spain. In: Sherif, Singh \& Al-Rashed, Groundwater Hydrology. Balkema, 2: 415-434.

Pulido-Bosch, A., Pulido-Leboeuf, P. and Gisbert, J. (2004). Pumping seawater from coastal aquifers for supplying desalination plants. Geological Acta, 2: 99-109.

Pulido Bosch, A. y Rodríguez Estrella, T. (2007). Los acuíferos costeros y el suministro de agua de mar a las plantas desaladoras. Las Aguas Subterráneas. Rev. Asoc. Enseñanza de Ciencias de la Tierra, Monográfico:: 274-279.

Pulido-Bosch, A., Delgado, J., Sola, F., Vallejos, A., Vicente, F., López-Sánchez, J.M. and Mallorquí, J. (2012). Identification of potential subsidence related to pumping in the Almería basin (SE Spain). Hydrological Processes, 26: 731-740.

Pulido-Bosch, A., Vallejos, A. and Sola, F. (2019). Methods to supply seawater to desalination plants along the Spanish Mediterranean coast and their associated issues. Environmental Earth Sciences, 78:322 https://doi.org/10.1007/ s12665-019-8298-9

Queralt i Creus, E. (2007). La Comunidad de Usuarios de Aguas del Valle Bajo y el Delta del Llobregat: 30 años de experiencia en la gestión de un acuífero costero. Boletín Geológico y Minero, 118 (Núm. Especial): 745-758.

Queralt, E., Bernat, X. and Custodio, E. (2020). Improving water supply security of the Lower Llobregat aquifers by 
integrating managed artificial recharge technologies into a conjunctive surface water and groundwater management scheme for Barcelona, Spain. Submitted to Water Resources Management.

Rabineau, M. (2001). Un modèle géométrique et stratigraphique des séquences de dépôt quaternaires sur la marge du Golfe du Lion: Enregistrement des cycles climatiques de 100.000 ans. PhD, University of Rennes 1: 1-480.

RAEMIA (2019). Recarga natural a los acuíferos, metodología y soporte de la isotopía del agua. Aplicación a la planificación hidrológica y conocimiento de las aguas subterráneas en España. Preparado por E. Custodio para UPC, Suez-España y Cetaqua, Barcelona: 1-800. OPC e-books. http://hdl.handle.net/XXX/Previsión diciembre 2019.

Renau-Pruñonosa, A., Morell, I. and Pulido-Velazquez, D. (2016). Methodology to analyse and assess pumping management strategies in coastal aquifers to avoid degradation due to seawater intrusion problems. Water $R e$ sources Management, 30(13): 4823-4837.

Rica Izquierdo, M. (2016). Análisis de las acciones colectivas en la gobernanza del agua subterránea en España. Tesis doctoral. Universidad Complutense de Madrid: 1-171. http://eprints.ucm.es/38258/1/T37444.pdf.

Rica, M., López-Gunn, E. and Llamas, M.R. (2012). An analytical framework on the emergence and evolution of collective action: an empirical case of Spanish groundwater user collective associations. Irrigation \& Drainage J., 61: 115-125.

Rodríguez-Estrella, T. and Pulido-Bosch, A. (2009). Methodologies for abstraction from coastal aquifers for supplying desalination plants in the south-east of Spain. Desalination, 249: 1088-1098.

SASMIE (2017) Salinización de las aguas subterráneas en los acuíferos costeros mediterráneos e insulares españoles. Preparado por E. Custodio para UPC, Suez Solutions y Cetaqua, Barcelona: 1-852, UPC e-books. http:// hdl.handle.net/2117/111515
Serra Raventós, J. y Verdaguer Andreu, A. 1983. La sedimentación holocena en el prodelta del Llobregat. En: A. Obrador (ed.), X Congreso Nacional de Sedimentología, Maó, 2: 47-51.

Simó, J.A., Gámez, D., Salvany, J.M., Vázquez-Suñé, E., Carrera, J., Barnolas, A. y Alcalá, F.J. (2005). Arquitectura de facies de los deltas cuaternarios del río Llobregat, BarceIona, España. Geogaceta, 38: 171-174.

SPA-15 (1975). Estudio científico de los recursos de agua en las Islas Canarias (SPA/69/5 15). Ministerio Obras Públicas. Dirección General Obras Hidráulicas. UNESCO. Las Palmas de Gran Canaria. Madrid. 4 Vol. + mapas.

TRLA (2001). Real Decreto Legislativo 1/2001, de 20 de julio, por el que se aprueba el texto refundido de la Ley deAguas. BOE 176, de 24 de julio de 2001: 26791-26817. http:// www.boe.es/buscar/doc.php?id=BOE-A-2001-14276.

TRLA (2003). Artículo 129. Modificación del texto refundido de la Ley de Aguas, aprobado por Real Decreto Legislativo $1 / 2001$, de 20 de julio, por la que se incorpora al derecho español, la Directiva 2000/60/CE, por la que se establece un marco comunitario de actuación en el ámbito de la política de aguas. Ley 62/2003, de 30 de diciembre, de medidas fiscales, administrativas y del orden social. BOE 313, de 31 de diciembre de 2003: 46874-46992.

Tulipano, L., Fidelibus, M.D. and Panagopoulos, A. (2003). Groundwater management of coastal karstic aquifers. European Commission, Cost Action 621 Final Report.

Vázquez-Suñè, E., Abarca. E., Carrera, J., Capino, D., Gámez, D., Simó, T., Batlle, F., Niñerola, J.M. and Ibáñez. X. 2006. Groundwater modelling as a tool for the European Water Framework Directive (WFD) application: The Llobregat case. Physics and Chemistry of the Earth, 31: 1015-1029.

Werner, A.D., Bakker, M., Post, V.E.A., Vandenbohede, A., Lu, C., Ataiee-Ashtani, B., Simmons, C.T. and Barry, D.A. (2013). Seawater intrusion processes, investigation and management: Recent advances and future challenges. Advances in Water Resources, 51: 3-26.

Recibido: julio 2019

Revisado: noviembre 2019

Aceptado: enero 2020

Publicado: marzo 2021 